\title{
Predicting the Binding Energies of the 1s Nuclides with High Precision, Based on Baryons which Are Yang-Mills Magnetic Monopoles
}

\author{
Jay R. Yablon \\ Schenectady, New York, USA \\ Email: jyablon@nycap.rr.com
}

Received March 22, 2013; revised April 24, 2013; accepted April 29, 2013

Copyright (C) 2013 Jay R. Yablon. This is an open access article distributed under the Creative Commons Attribution License, which permits unrestricted use, distribution, and reproduction in any medium, provided the original work is properly cited.

\begin{abstract}
In an earlier paper, the author employed the thesis that baryons are Yang-Mills magnetic monopoles and that proton and neutron binding energies are determined based on their up and down current quark masses to predict a relationship among the electron and up and down quark masses within experimental errors and to obtain a very accurate relationship for nuclear binding energies generally and for the binding of ${ }^{56} \mathrm{Fe}$ in particular. The free proton and neutron were understood to each contain intrinsic binding energies which confine their quarks, wherein some or most (never all) of this energy is released for binding when they are fused into composite nuclides. The purpose of this paper is to further advance this thesis by seeing whether it can explain the specific empirical binding energies of the light 1s nuclides, namely, ${ }^{2} \mathrm{H},{ }^{3} \mathrm{H},{ }^{3} \mathrm{He}$ and ${ }^{4} \mathrm{He}$, with high precision. As the method to achieve this, we show how these $1 \mathrm{~s}$ binding energies are in fact the components of inner and outer tensor products of Yang-Mills matrices which are implicit in the expressions for these intrinsic binding energies. The result is that the binding energies for the ${ }^{4} \mathrm{He},{ }^{3} \mathrm{He}$ and ${ }^{3} \mathrm{H}$ nucleons are respectively, independently, explained to less than four parts in one million, four parts in 100,000, and seven parts in one million, all in AMU. Further, we are able to exactly relate the neutron minus proton mass difference to a function of the up and down current quark masses, which in turn enables us to explain the ${ }^{2} \mathrm{H}$ binding energy most precisely of all, to just over 8 parts in ten million. These energies have never before been theoretically explained with such accuracy, which leads to the conclusion that the underlying thesis provides the strongest theoretical explanation to date of what baryons are, and of how protons and neutrons confine their quarks and bind together into composite nuclides. As is also reviewed in Section 9, these results may lay the foundation for more easily catalyzing nuclear fusion energy release.
\end{abstract}

Keywords: Nuclides; Binding Energy; Deuteron; Triton; Helion; Alpha; Alpha Decay; Beta Decay; Yang-Mills; Magnetic Monopoles; Solar Fusion; Nuclear Fusion; Confinement

\section{Introduction: Summary Review of the Thesis that Baryons Are Yang-Mills Magnetic Monopoles with Binding Energies Based on Their Current Quark Masses}

In an earlier paper [1], the author developed the thesis that magnetic monopole densities which come into existence in a non-Abelian Yang-Mills gauge theory of non-commuting vector gauge boson fields $G^{\mu}$ are synonymous with baryon densities. That is, baryons, including the protons and neutrons which form the vast preponderance of matter in the universe, are Yang-Mills magnetic monopoles. Conversely, magnetic monopoles, long pursued since the time of Maxwell, have always been hiding in plain sight, in Yang-Mills incarnation, as baryons, and especially, as protons and neutrons.

Maxwell's equations themselves provide the theoretical foundation for this thesis, because if one starts with the classical electric charge and magnetic monopole field equations (respectively, (2.1) and (2.2) of [1]):

$$
\begin{aligned}
J^{v} & =\partial_{\mu} F^{\mu v}=\partial_{\mu} D^{[\mu} G^{v]} \\
& =\left(g^{\mu v} \partial_{\sigma} D^{\sigma}-\partial^{\mu} D^{v}\right) G_{\mu} \\
P^{\sigma \mu v} & =\partial^{\sigma} F^{\mu v}+\partial^{\mu} F^{v \sigma}+\partial^{v} F^{\sigma \mu}
\end{aligned}
$$

$\left(D^{\mu} \equiv \partial^{\mu}-i G^{\mu}\right)$ and combines the magnetic charge Equation (1.2) with a Yang-Mills (non-Abelian) field strength tensor $F^{\mu v}$ which, like $G^{\mu}$ is an $N \times N$ ma- 
trix for a simple gauge group $S U(N)((2.3)$ of [1]):

$$
\begin{aligned}
F^{\mu \nu} & =\partial^{\mu} G^{v}-\partial^{v} G^{\mu}-i\left[G^{\mu}, G^{v}\right] \\
& =D^{\mu} G^{v}-D^{v} G^{\mu}=D^{[\mu} G^{v]}
\end{aligned}
$$

one immediately comes upon the non-vanishing magnetic monopole ((2.4) of [1]):

$$
\begin{aligned}
P^{\sigma \mu v} & =-i\left(\partial^{\sigma}\left[G^{\mu}, G^{v}\right]\right. \\
& \left.+\partial^{\mu}\left[G^{v}, G^{\sigma}\right]+\partial^{v}\left[G^{\sigma}, G^{\mu}\right]\right)
\end{aligned}
$$

The question then becomes whether such magnetic monopoles (1.4) actually do exist in the material universe, and if so, in what form. The thesis developed in [1] is not only that these magnetic monopoles do exist, but that they permeate the material universe in the form of baryons, especially as the protons and neutrons observed everywhere and anywhere that matter exists.

Of course, t'Hooft [2] and Polyakov [3] realized several decades ago that non-Abelian gauge theories lead to non-vanishing magnetic monopoles. But their monopoles have very high energies which make them not suitable for being baryons such as protons and neutrons. Following t'Hooft, the author in [1] does make use of the t'Hooft monopole Lagrangian from (2.1) of [2] to calculate the energies of these magnetic monopoles (1.4). But whereas t'Hooft introduces an ansatz about the radial behavior of the gauge bosons $G^{\mu}$, the author instead makes use of a Gaussian ansatz borrowed from Equation (14) of Ohanian's [4] for the radial behavior of fermions. Moreover, the fermions for which this ansatz is employed enter on the very solid foundation of taking the inverse $G_{v} \equiv I_{\sigma v} J^{\sigma}$ of Maxell's charge Equation (1.1) (essentially calculating the configuration space inverse $\left.\left(g^{\mu v} \partial_{\sigma} D^{\sigma}-\partial^{\mu} D^{v}\right)^{-1}\right)$, and then combining this with the relationship $J^{\mu}=\bar{\psi} \gamma^{\mu} \psi$ that emerges from satisfying the charge conservation (continuity) equation $\partial_{\mu} J^{\mu}=0$ in Dirac theory. Specifically, it was found that in the low-perturbation limit, magnetic monopoles (1.4) can be re-expressed as a three-fermion system ((3.12) of [1]):

$$
\begin{aligned}
P^{\sigma \mu v} & =-2\left(\partial^{\sigma} \frac{\bar{\psi}_{(1)} \sigma^{\mu_{\vee} v} \psi_{(1)}}{" \rho_{(1)}-m_{(1)} "}\right. \\
& \left.+\partial^{\mu} \frac{\bar{\psi}_{(2)} \sigma^{v_{v} \sigma} \psi_{(2)}}{" \rho_{(2)}-m_{(2)} "}+\partial^{v} \frac{\bar{\psi}_{(3)} \sigma^{\sigma_{v} \mu} \psi_{(3)}}{" \rho_{(3)}-m_{(3)} "}\right)
\end{aligned}
$$

Above, $\psi_{(i)} ; i=1,2,3$ are three distinct Dirac spinor wavefunctions which emerge following three distinct substitutions of $G_{v}=I_{\sigma v} J^{\sigma}=I_{\sigma v} \bar{\psi} \gamma^{\sigma} \psi$-which captures the inverse of Maxwell's charge Equation (1.1) combined with Dirac theory-into the (1.4) magnetic monopole which utilizes the Yang-Mills field strength
(1.3) in combination with Maxwell's magnetic monopole Equation (1.2). The detailed derivation of (1.5) from (1.4) also makes use of Sections 6.2, 6.14 and 5.5 of [5] pertaining to Compton scattering and the fermion completeness relation, and carefully accounts for mass degrees of freedom as between fermions and bosons. The quoted denominators " $p_{(i)}-m_{(i)}$ " and "quasi commutators" $\sigma^{\mu_{v} v} \equiv \frac{i}{2}\left[\gamma^{\mu}{ }_{v} \gamma^{v}\right]$ in the above make use of a compact notation developed and explained in Section 3 of [1], see specifically (3.9) and (3.10) therein.

Then, via Fermi-Dirac Exclusion, the author employed the QCD color group $S U(3)_{C}$ to require that each of the three $\psi_{(i)}$ be $S U(3)_{C}$ vectors in distinct quantum color eigenstates R, G, B, which then leads in (5.5) of [1] to a magnetic monopole:

$$
\begin{aligned}
\operatorname{Tr} P^{\sigma \mu v} & =-2\left(\partial^{\sigma} \frac{\bar{\psi}_{R} \sigma^{\mu_{\vee} v} \psi_{R}}{" \rho_{R}-m_{R} "}\right. \\
& \left.+\partial^{\mu} \frac{\bar{\psi}_{G} \sigma^{v_{v} \sigma} \psi_{G}}{" \rho_{G}-m_{G} "}+\partial^{v} \frac{\bar{\psi}_{B} \sigma^{\sigma_{\vee} \mu} \psi_{B}}{" \rho_{B}-m_{B} "}\right)
\end{aligned}
$$

This is similar to (1.5) but for the emergence of the trace. Associating each color with the spacetime index in the related $\partial^{\sigma}$ operator, i.e., $\sigma \sim R, \mu \sim G$ and $v \sim B$, and keeping in mind that $\operatorname{Tr} P^{\sigma \mu \nu}$ is antisymmetric in all spacetime indexes, we express this antisymmetry with wedge products as $\sigma \wedge \mu \wedge v \sim R \wedge G \wedge B$. So the natural antisymmetry of a magnetic monopole $P^{\sigma \mu v}$ leads straight to the required antisymmetric color singlet wavefunction $R[G, B]+G[B, R]+B[R, G]$ for a baryon. Indeed, in hindsight, this antisymmetry together with three vector indexes to accommodate three vector current densities and the three additive terms in the $P^{\sigma \mu v}$ of (1.2) should have been a tip-off that magnetic monopoles would naturally make good baryons. Further, upon integration over a closed surface via Gauss'/Stokes' theorem, magnetic monopole (1.6) is shown to emit and absorb color singlets with the symmetric color wavefunction $\bar{R} R+\bar{G} G+\bar{B} B$ expected of a meson. And, in Section 1 of [1], it was shown how magnetic monopoles naturally contain their gauge fields in non-Abelian gauge theory via the differential forms relationship $d d=0$ for precisely the same reasons rooted in spacetime geometry that magnetic monopoles do not exist at all in Abelian gauge theory. Thus, QCD itself deductively emerges from the thesis that baryons are Yang-Mills magnetic monopoles, and we began to associate monopole (1.6) with a baryon.

It was then shown in Sections 6 through 8 of [1] that these $S U(3)$ monopoles may be made topologically stable by symmetry breaking from larger $S U(4)$ gauge groups which yield the baryon and electric charge quantum numbers of a proton and neutron. Specifically, the topological stability of these magnetic monopoles was estab- 
lished in Sections 6 and 8 of [1] based on Cheng and $\mathrm{Li}$ [6] at 472-473 and Weinberg [7] at 442. The proton and neutron are developed as particular types of magnetic monopole in Section 7 of [1] making use of $S U(4)$ gauge groups for baryon minus lepton number $B-L$ based on Volovok's [8], Section 12.2.2. The spontaneous symmetry breaking of these $S U(4)$ gauge groups is then fashioned on Georgi-Glashow's SU(5) GUT model [9] reviewed in detail in Section 8 of [1].

By then employing the earlier-referenced "Gaussian ansatz" from Ohanian's [4], namely ((9.9) of [1]):

$$
\psi(r)=u(p)\left(\pi \lambda^{2}\right)^{-\frac{3}{4}} \exp \left(-\frac{1}{2} \frac{\left(r-r_{0}\right)^{2}}{\lambda^{2}}\right)
$$

for the radial behavior of the fermion wavefunctions, together with the t'Hooft monopole Lagrangian from (2.1) of [2] (see (9.2) of [1]) it became possible to analytically calculate the energies of these Yang-Mills magnetic monopoles (1.6) following their development into topologically stable protons and neutrons.

Specifically, in Sections 11 and 12 of [1], the author used the pure gauge field terms $\mathfrak{L}_{\text {gauge }}$ of the t'Hooft monopole Lagrangian to specify the energy of the YangMills magnetic monopoles, exclusive of the vacuum $\Phi$, via (11.7) of [1]:

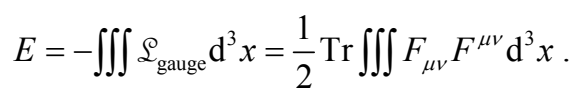

We then made use in (1.8) of field strength tensors for protons and neutrons developed via Gauss'/Stokes' theorem from (1.6) in (11.3) and (11.4) of [1], respectively:

$$
\begin{aligned}
& \operatorname{Tr} F_{\mathrm{P}}^{\mu \nu}= \\
& -i\left(\frac{\bar{\psi}_{d}\left[\gamma^{\mu}{ }_{\vee} \gamma^{v}\right] \psi_{d}}{" \rho_{d}-m_{d} "}+2 \frac{\bar{\psi}_{u}\left[\gamma^{\mu}{ }_{\vee} \gamma^{v}\right] \psi_{u}}{" \rho_{u}-m_{u} "}\right) \\
& \operatorname{Tr} F_{\mathrm{N}}^{\mu \nu}= \\
& -i\left(\frac{\bar{\psi}_{u}\left[\gamma^{\mu}{ }_{v} \gamma^{v}\right] \psi_{u}}{" \rho_{u}-m_{u} "}+2 \frac{\bar{\psi}_{d}\left[\gamma^{\mu}{ }_{v} \gamma^{v}\right] \psi_{d}}{" \rho_{d}-m_{d} "}\right)
\end{aligned}
$$

where $\psi_{u}$ and $\psi_{d}$ are Dirac wavefunctions for up and down quarks, to deduce three relationships which yielded remarkable concurrence with empirical data.

First, we found in (11.22) of [1] that the electron mass is related to up and down quark masses according to:

$$
m_{e}=0.510998928 \mathrm{MeV}=3\left(m_{d}-m_{u}\right) /(2 \pi)^{\frac{3}{2}},
$$

where the divisor $(2 \pi)^{\frac{3}{2}}$ results as a natural consequence of the three-dimensional integration (1.8) when the Gaussian ansatz for fermions is specified as in (1.7), and where the wavelengths in (1.7) are taken to be related to the quark masses via the de Broglie relation $\lambda=\hbar / m c$.

Second and third, we found in (12.12) and (12.13) of [1] that if one postulates the current mass of the up quark to be equal to the deuteron $\left({ }^{2} \mathrm{H}\right.$ nucleus) binding energy based on 1) empirical concurrence within experimental errors and 2) regarding nucleons to be resonant cavities with binding energies determined in relation to their up and down current quark masses, then the proton and neutron each possess respective intrinsic, latent binding energies B (i.e., energies intrinsically available for nuclear binding):

$$
\begin{aligned}
\mathrm{B}_{\mathrm{P}} & =2 m_{u}+m_{d}-\left(m_{d}+4 \sqrt{m_{u} m_{d}}+4 m_{u}\right) /(2 \pi)^{\frac{3}{2}} \\
& =7.640679 \mathrm{MeV} \\
\mathrm{B}_{\mathrm{N}} & =2 m_{d}+m_{u}-\left(m_{u}+4 \sqrt{m_{u} m_{d}}+4 m_{d}\right) /(2 \pi)^{\frac{3}{2}} \\
& =9.812358 \mathrm{MeV}
\end{aligned}
$$

So for a nucleus with an equal number of protons and neutrons, the average binding energy per nucleon is predicted to be $8.726519 \mathrm{MeV}$. Not only does this explain why a typical nucleus beyond the very lightest (which we shall be studying in detail here) has a binding energy in exactly this vicinity (see Figure 1 below), but when this is applied to ${ }^{56} \mathrm{Fe}$ with 26 protons and 30 neutronswhich has the distinction of using a higher percentage of this available binding energy than any other nuclide - we see that the latent available binding energy is predicted to be ((12.14) of [1]):

$$
\begin{aligned}
\mathrm{B}\left(\mathrm{Fe}^{56}\right) & =26 \times 7.640679 \mathrm{MeV} \\
& +30 \times 9.812358 \mathrm{MeV} \\
& =493.028394 \mathrm{MeV}
\end{aligned}
$$

This contrasts remarkably with the observed ${ }^{56} \mathrm{Fe}$ binding energy of $492.253892 \mathrm{MeV}$. That is, precisely $99.8429093 \%$ of the available binding energy predicted by this model of nucleons as Yang-Mills magnetic monopoles goes into binding together the ${ }^{56} \mathrm{Fe}$ nucleus, with a small $0.1570907 \%$ balance reserved for confining quarks within each nucleon. This means while quarks are very much freer in the nucleons of ${ }^{56} \mathrm{Fe}$ than in free nucleons (which also appears to explain the "first EMC effect" [10]), their confinement is never fully overcome. Confinement bends but never breaks. Quarks step back from the brink of becoming de-confined in ${ }^{56} \mathrm{Fe}$ as one moves to even heavier nuclides, and remain confined no matter what the nuclide. Iron-56 thus sits at the theoretical crossroads of fission, fusion and confinement.

This thesis that protons and neutrons are resonant cavities which emit and absorb energies that directly manifest their current quark masses will be central to the 


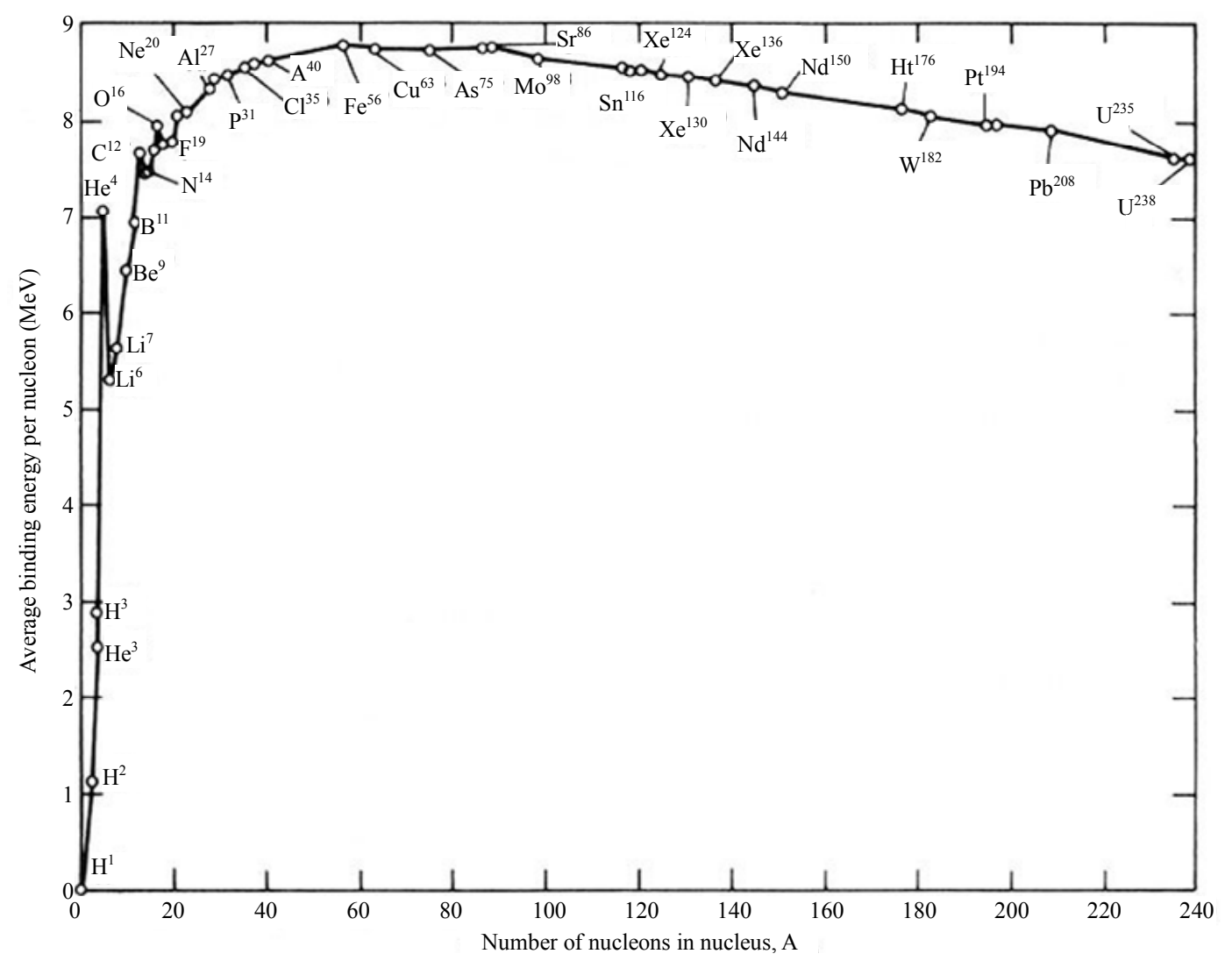

Figure 1. The empirical binding energy per nucleon of various nuclides.

development of this paper. The foregoing (1.12) through (1.14) provide strong preliminary confirmation of this thesis, as well as of the underlying thesis that baryons are Yang-Mills magnetic monopoles. In this paper, we shall show how the observed binding energies of the $1 \mathrm{~s}$ nuclides, namely of ${ }^{2} \mathrm{H},{ }^{3} \mathrm{H},{ }^{3} \mathrm{He}$ and ${ }^{4} \mathrm{He}$, as well as the observed neutron minus proton mass difference, provide further compelling confirmation of the thesis that baryons are Yang-Mills magnetic monopoles which bind at energies which directly reflect the current quark masses they contain.

In simple summation: with a non-Abelian Yang-Mills field strength (1.3), Yang Mills magnetic monopole baryons result from simply combining Maxwell's classical electric (1.1) and magnetic (1.2) charge equations together into a single equation, making use of Dirac's $J^{\mu}=\bar{\psi} \gamma^{\mu} \psi$ based on charge continuity, and imposing Fermi-Dirac $S U(3)_{C}$ Exclusion on the fermions of the resulting three-fermion monopole system. No further ingredients or assumptions are required, and all of these ingredients being so-combined in novel fashion are among the undisputed, uncontroversial bedrock foundations of modern physics. The Gaussian ansatz (1.7) enables the energy (1.8) to be analytically calculated, the mass relation (1.11) naturally emerges, and once we further apply the resonant cavity thesis, the resulting ener- gies turn out to match up remarkably well with nuclear binding energies.

In even simpler summation: Maxwell's Equations (1.1), (1.2) themselves, combined together into one equation using non-Abelian gauge fields (1.3), taken together with Dirac theory and Fermi-Dirac Exclusion, are the governing equations of nuclear physics, insofar as nuclear physics centers around the study of protons and neutrons and how they bind and interact, and given that we were able to show in [1] that protons and neutrons are particular types of Yang-Mills magnetic monopoles. This theory is thus extremely conservative, based on combining together unquestionable foundational physics principles.

In essence, the purpose of this paper is to further develop the results from [1] into a theory of nuclear binding which we confirm by predicting the binding energies of the $1 \mathrm{~s}$ nuclides as well as the neutron minus proton mass difference with very high precision, each on the order of parts per million.

\section{Structured Outline of the Contents of This Paper}

In deriving the empirically-accurate binding energy relationships (1.12) through (1.14) there is an aspect of (1.8) which, when carefully considered, requires us to amend 
the Lagrangian in (1.8) in a slight but important way. This amendment, developed in Section 3, will reveal that the latent binding energies (1.12) and (1.13) actually employ the inner and outer tensor products of two $3 \times 3$ $S U(3)$ matrices, one for protons, and one for neutrons. These matrices, and their inner and outer products, will be critical to the methodological development thereafter.

In section 4 we lay the foundation for being able to derive the binding energies of the $1 \mathrm{~s}$ nuclides using the earlier-discussed postulate that the mass of the up quark is equal to the deuteron $\left({ }^{2} \mathrm{H}\right.$ nucleus) binding energy, and the thesis extrapolated from this that the binding energies of nuclides generally are direct functions of the current quark masses which their nucleons contain. Specifically, in (4.9) through (4.11) infra, we develop two tensor outer products and their components which will be critical ingredients for expressing 1s binding energies as functions of up and down current quark masses.

Section 5 shows how this binding energy thesis leads directly to a theoretical expression for the ${ }^{4} \mathrm{He}$ alpha binding energy which matches empirical data to less than 3 parts in 1 million AMU. Exploring the meaning of this result, we see that this binding energy together with that of the ${ }^{2} \mathrm{H}$ deuteron are actually components of a $(3 \times 3) \times$ $(3 \times 3)$ fourth rank Yang Mills tensor of which the ${ }^{2} \mathrm{H}$ and ${ }^{4} \mathrm{He}$ binding energies merely two samples. Thus, we are motivated to think about binding energies generally as components of Yang-Mills tensors. So the method for characterizing binding energies is one of trying to match up empirical binding energies with various expressions which emerge from, or are components of, these YangMills tensors. In Section 6, we similarly obtain a theoretical expression for ${ }^{3} \mathrm{He}$ helion binding to just under 4 parts in 100,000 AMU as well as its characterization in terms of these Yang-Mills tensors.

Developing a similar expression for the ${ }^{3} \mathrm{H}$ triton to what ends up being just over three parts in one million AMU turns out to be less straightforward than for any of ${ }^{2} \mathrm{H},{ }^{3} \mathrm{He}$ and ${ }^{4} \mathrm{He}$, and requires us to work with mass excess rather than binding energy. However, a bonus is that in the process, we are also motivated to derive an expression for the neutron minus proton mass difference accurate to just over 7 parts in ten million AMU. To maintain clarity and focus on the underlying research ideas, these results are summarized in Section 7, while their detailed derivation is presented in the Appendix.

Section 8 aggregates the results of Sections 5 through 7 , and couches them all in terms of mass excess rather than binding energy. In this form, it becomes more straightforward to study nuclear fusion processes involving these $1 \mathrm{~s}$ nuclides.

Section 9 makes use of the mass excess results from Section 8, and shows how these can be combined to ex- press the approximately $26.73 \mathrm{MeV}$ of energy known to be released during the solar fusion cycle $4 \cdot{ }_{1}^{1} \mathrm{H}+2 e^{-} \rightarrow$ ${ }_{2}^{4} \mathrm{He}+2 v+$ Energy entirely in terms of the up, down and electron fermion masses. This highlights not only the accuracy of the results for ${ }^{2} \mathrm{H},{ }^{3} \mathrm{H},{ }^{3} \mathrm{He}$ and ${ }^{4} \mathrm{He}$ binding energies and the neutron minus proton mass difference, but it establishes the approach one would use to do the same for other types of nuclear fusion, and for fission reactions. And, it vividly confirms the thesis that fusion and fission and binding energies are directly based on the masses of the quarks which are contained in protons and neutrons, regarded as resonant cavities.

But perhaps the most important consequence of the development in Section 9 is technological, because the possibility is developed via this "resonant cavity" analysis that by bathing a store of hydrogen in gamma radiation at certain specified, discrete frequencies which are also defined functions of the up and down quark masses, one can catalyze nuclear fusion and perhaps develop more effective ways to practically exploit the promise of nuclear fusion energy release.

In Section 10, we take a closer look at experimental errors that still do reside in the results for ${ }^{3} \mathrm{H},{ }^{3} \mathrm{He}$ and ${ }^{4} \mathrm{He}$ binding and the neutron minus proton mass difference, generally at parts per $10^{5}, 10^{6}$ or $10^{7}$ AMU. We explain why the original postulate identifying the up quark mass exactly with the ${ }^{2} \mathrm{H}$ deuteron binding energy should be modified into the substitute postulate that the theoretical neutron minus proton mass difference is an exact relationship, and why the equality of the up quark mass and the deuteron binding energy is simply a very close approximation (to just over 8 parts in ten million) rather than an exact relationship. We then are required to adjust (recalibrate) all of the prior numeric mass and energy calculations accordingly, by about parts per million. As a by-product, the up and down quark masses become known with the same degree of experimental precision as the electron rest mass and the neutron minus proton mass difference, to ten decimal places in $A M U$.

Section 11 concludes by summarizing and consolidateing these results, laying out most compactly in Figure 11, infra, how the thesis that baryons are Yang-Mills magnetic monopoles which fuse at binding energies reflective of their current quark masses can be used to predict the binding energies of the ${ }^{4} \mathrm{He}$ alpha to less than four parts in one million, of the ${ }^{3} \mathrm{He}$ helion to less than four parts in 100,000 , and of the ${ }^{3} \mathrm{H}$ triton to less than seven parts in one million, all in AMU. And of special import, by exactly relating the neutron minus proton mass difference to a function of the up and down quark masses, we are enabled to predict the binding energy for the ${ }^{2} \mathrm{H}$ deuteron most precisely of all, to just over 8 parts in ten million.

What renders this work novel is 1) that the $1 \mathrm{~s}$ light 
nuclide binding energies and the neutron minus proton mass difference do not appear to have ever before been theoretically explained with such accuracy; 2) the degree to which this accuracy confirms that baryons are YangMills magnetic monopoles with binding energies which are components of a Yang-Mills tensor and which are directly related to current quark masses contained in these baryons; 3 ) the finding that nuclear physics appears to be grounded in unquestionable conservative physics principles, governed by simply combining Maxwell's two classical equations into one equation using Yang-Mills gauge fields in view of Dirac theory and Fermi-Dirac Exclusion for fermions; and 4) the prospect of perhaps improving nuclear fusion technology by applying suitably-chosen resonances of gamma radiation for catalysis.

\section{The Lagrangian of Nuclear Binding Energies}

The t'Hooft magnetic monopole Lagrangian used in (1.8), because of suppression of the Yang-Mills matrix indexes, actually has an ambiguous mathematical meaning, and can be either an ordinary (inner product) matrix multiplication, or a tensor (outer) product. The outer product is the most general bilinear operation that can be performed on $F_{\mu \nu} F^{\mu v}$, while the inner product represents a contraction of the outer product which reduces the YangMills rank by 2 . When carefully considered, this provides an opportunity for developing a nuclear Lagrangian based on the t'Hooft's original development [2] of YangMills magnetic monopoles.

If we know that $\frac{1}{4} F_{a \mu \nu} F_{a}^{\mu v}=\frac{1}{2} F_{\mu v} F^{\mu v}$ as we do from the terms in (11.7) of [1] omitted from (1.8) above, and given that $\operatorname{Tr} T^{i} T^{j}=\frac{1}{2} \delta^{i j}$, then with explicit indexes $A, B, C, D=1,2,3$ for the $3 \times 3$ Yang-Mills matrices of the $S U(3)_{C^{\prime}}$ isospin-modified color group developed in Section 8 of [1], an explicit appearance of Yang-Mills indexes would cause (1.8) to be written as:

$$
\begin{aligned}
E & =-\iiint \mathfrak{S}_{\text {gauge }} \mathrm{d}^{3} x=\frac{1}{2} \operatorname{Tr} \iiint F_{\mu \nu} F^{\mu v} \mathrm{~d}^{3} x=\frac{1}{2} \operatorname{Tr} \iiint F_{\mu v A B} F_{B D}^{\mu v} \mathrm{~d}^{3} x \\
& =\frac{1}{2} \operatorname{Tr} \iiint F_{A B} \cdot F_{B D} \mathrm{~d}^{3} x=\frac{1}{2} \iiint F_{A B} \cdot F_{B A} \mathrm{~d}^{3} x
\end{aligned}
$$

where $F \cdot F \equiv F_{\mu v} F^{\mu v}$ suppresses spacetime indexes to focus attention on contractions of Yang-Mills indexes. In the fourth and fifth terms above, there is a contraction over the inner " $B$ " index, which means that $F_{A B} \cdot F_{B D}$ is an inner product formed with ordinary matrix multiplication, and is a contraction over inner indexes of the fourth rank $(3 \times 3 \times 3 \times 3)$ outer product $F_{\mu v} \otimes F^{\mu v}=$ $F_{A B} \cdot F_{C D}$ down to rank two. In the sixth, final term, we write $\operatorname{Tr} F_{A B} \cdot F_{B D}=F_{A B} \cdot F_{B A}$ via a second " $A$ " index contraction.

We point this out because (1.12) through (1.14) which successfully match empirical nuclear binding data, embody not only (3.1), but also an outer product $F_{A B} \cdot F_{C D}$, that is, (carefully contrast Yang-Mills indexes between the final terms in (3.1), (3.2)):

$$
\begin{aligned}
E & =-\iiint \mathfrak{\varrho}_{\text {gauge }} \mathrm{d}^{3} x=\frac{1}{2} \operatorname{Tr} \iiint F_{\mu \nu} \otimes F^{\mu v} \mathrm{~d}^{3} x=\frac{1}{2} \operatorname{Tr} \iiint F_{\mu \nu A B} F_{C D}^{\mu v} \mathrm{~d}^{3} x \\
& =\frac{1}{2} \operatorname{Tr} \iiint F_{A B} \cdot F_{C D} \mathrm{~d}^{3} x=\frac{1}{2} \iiint F_{A A} \cdot F_{B B} \mathrm{~d}^{3} x
\end{aligned}
$$

here, in the final terms, we use $\operatorname{Tr} F_{A B} \cdot F_{C D}=F_{A A} \cdot F_{B B}$, as opposed to $\operatorname{Tr} F_{A B} \cdot F_{B D}=F_{A B} \cdot F_{B A}$. This highlights the notational ambiguity in (1.8) as well as the difference between the outer $\otimes$ and inner matrix products.

Now, in general, the trace of a product of two square matrices is not the product of traces. The only circumstance in which "trace of a product" equals "product of traces" is when one forms a tensor outer product using:

$$
\operatorname{Tr}(A \otimes B)=\operatorname{Tr}(A) \operatorname{Tr}(B) .
$$

Specifically, to obtain the terms $m_{d}+4 \sqrt{m_{u} m_{d}}+4 m_{u}$ and $m_{u}+4 \sqrt{m_{u} m_{d}}+4 m_{d}$ in (1.12) and (1.13) (and also
(12.4) and (12.5) of [1] which erroneously applied (3.2), (3.3) rather than (3.1) because of this ambiguity), we must use (3.2), while to obtain $2 m_{u}+m_{d}$ and $2 m_{d}+m_{u}$ in (1.12) and (1.13), we instead must use (3.1). So (1.12) and (1.13) are formed by a linear combination of both inner and outer products. And because (1.12) and (1.13) predict binding energies per nucleon in the range of 8.7 $\mathrm{MeV}$ and yield an extremely close match to ${ }^{56} \mathrm{Fe}$ binding energies, nature herself appears to be telling us that we need to combine inner and outer products in this way in order to match up with empirical data. This, in turn, gives us important feedback for how to construct our Lagrangian to match the empirical data. 
To see this most vividly, we start with (11.8) and (11.9) from [1]:

$$
\begin{aligned}
& E_{\mathrm{P}}=-\frac{1}{2} \iiint\left(\frac{\bar{\psi}_{d}\left[\gamma^{\mu}{ }_{v} \gamma^{v}\right] \psi_{d}}{" \rho_{d}-m_{d} "}+2 \frac{\bar{\psi}_{u}\left[\gamma^{\mu}{ }_{v} \gamma^{v}\right] \psi_{u}}{" \rho_{u}-m_{u} "}\right) \times\left(\frac{\bar{\psi}_{d}\left[\gamma_{\mu \vee} \gamma_{v}\right] \psi_{d}}{" \rho_{d}-m_{d} "}+2 \frac{\bar{\psi}_{u}\left[\gamma_{\mu \vee} \gamma_{v}\right] \psi_{u}}{" \rho_{u}-m_{u} "}\right) \mathrm{d}^{3} x \\
& E_{\mathrm{N}}=-\frac{1}{2} \iiint\left(\frac{\bar{\psi}_{u}\left[\gamma^{\mu}{ }_{\vee} \gamma^{v}\right] \psi_{u}}{" \rho_{u}-m_{u} "}+2 \frac{\bar{\psi}_{d}\left[\gamma^{\mu}{ }_{\vee} \gamma^{v}\right] \psi_{d}}{" \rho_{d}-m_{d} "}\right) \times\left(\frac{\bar{\psi}_{u}\left[\gamma_{\mu \vee} \gamma_{v}\right] \psi_{u}}{" \rho_{u}-m_{u} "}+2 \frac{\bar{\psi}_{d}\left[\gamma_{\mu \vee} \gamma_{v}\right] \psi_{d}}{" \rho_{d}-m_{d} "}\right) \mathrm{d}^{3} x
\end{aligned}
$$

Using these in (3.1) and (3.2) following the development in Section 11 and (12.12) and (12.13) of [1], we can reproduce Equations (1.12) and (1.13) for the empiri- cally-accurate latent binding energies of a proton and neutron using linear combinations of inner and outer Yang-Mills matrix products, respectively, as follows:

$$
\begin{aligned}
& \mathrm{B}_{\mathrm{P}}=\Sigma E_{\mathrm{P}}-E_{\mathrm{P}}=\frac{1}{2} \operatorname{Tr} \iiint\left((2 \pi)^{\frac{3}{2}} F_{\mathrm{P} \mu v} F_{\mathrm{P}}^{\mu v}-F_{\mathrm{P} \mu v} \otimes F_{\mathrm{P}}^{\mu v}\right) \mathrm{d}^{3} x=\frac{1}{2} \operatorname{Tr} \iiint\left((2 \pi)^{\frac{3}{2}} F_{\mathrm{P} A B} \cdot F_{\mathrm{P} B D}-F_{\mathrm{P} A B} \cdot F_{\mathrm{P} C D}\right) \mathrm{d}^{3} x \\
& =\frac{1}{2} \iiint\left((2 \pi)^{\frac{3}{2}} F_{\mathrm{P} A B} \cdot F_{\mathrm{P} B A}-F_{\mathrm{P} A A} \cdot F_{\mathrm{P} B B}\right) \mathrm{d}^{3} x=2 m_{u}+m_{d}-\frac{1}{(2 \pi)^{\frac{3}{2}}}\left(m_{d}+4 \sqrt{m_{u} m_{d}}+4 m_{u}\right) \\
& =\operatorname{Tr}\left[\left(\begin{array}{ccc}
\sqrt{m_{d}} & 0 & 0 \\
0 & \sqrt{m_{u}} & 0 \\
0 & 0 & \sqrt{m_{u}}
\end{array}\right)\left(\begin{array}{ccc}
\sqrt{m_{d}} & 0 & 0 \\
0 & \sqrt{m_{u}} & 0 \\
0 & 0 & \sqrt{m_{u}}
\end{array}\right)-\frac{1}{(2 \pi)^{\frac{3}{2}}}\left(\begin{array}{ccc}
\sqrt{m_{d}} & 0 & 0 \\
0 & \sqrt{m_{u}} & 0 \\
0 & 0 & \sqrt{m_{u}}
\end{array}\right) \otimes\left(\begin{array}{ccc}
\sqrt{m_{d}} & 0 & 0 \\
0 & \sqrt{m_{u}} & 0 \\
0 & 0 & \sqrt{m_{u}}
\end{array}\right)\right] \\
& =9.356376 \mathrm{MeV}-1.715697 \mathrm{MeV}=7.640679 \mathrm{MeV} \\
& \mathrm{B}_{\mathrm{N}}=\Sigma E_{\mathrm{N}}-E_{\mathrm{N}}=\frac{1}{2} \operatorname{Tr} \iiint\left((2 \pi)^{\frac{3}{2}} F_{\mathrm{N} \mu v} F_{\mathrm{N}}^{\mu v}-F_{\mathrm{N} \mu v} \otimes F_{\mathrm{N}}^{\mu v}\right) \mathrm{d}^{3} x=\frac{1}{2} \operatorname{Tr} \iiint\left((2 \pi)^{\frac{3}{2}} F_{\mathrm{N} A B} \cdot F_{\mathrm{N} B D}-F_{\mathrm{N} A B} \cdot F_{\mathrm{N} C D}\right) \mathrm{d}^{3} x \\
& =\frac{1}{2} \iiint\left((2 \pi)^{\frac{3}{2}} F_{\mathrm{N} A B} \cdot F_{\mathrm{N} B A}-F_{\mathrm{N} A A} \cdot F_{\mathrm{N} B B}\right) \mathrm{d}^{3} x=2 m_{d}+m_{u}-\frac{1}{(2 \pi)^{\frac{3}{2}}}\left(m_{u}+4 \sqrt{m_{u} m_{d}}+4 m_{d}\right) \\
& =\operatorname{Tr}\left[\left(\begin{array}{ccc}
\sqrt{m_{u}} & 0 & 0 \\
0 & \sqrt{m_{d}} & 0 \\
0 & 0 & \sqrt{m_{d}}
\end{array}\right)\left(\begin{array}{ccc}
\sqrt{m_{u}} & 0 & 0 \\
0 & \sqrt{m_{d}} & 0 \\
0 & 0 & \sqrt{m_{d}}
\end{array}\right)-\frac{1}{(2 \pi)^{\frac{3}{2}}}\left(\begin{array}{ccc}
\sqrt{m_{u}} & 0 & 0 \\
0 & \sqrt{m_{d}} & 0 \\
0 & 0 & \sqrt{m_{d}}
\end{array}\right) \otimes\left(\begin{array}{ccc}
\sqrt{m_{u}} & 0 & 0 \\
0 & \sqrt{m_{d}} & 0 \\
0 & 0 & \sqrt{m_{d}}
\end{array}\right)\right] \\
& =12.039054 \mathrm{MeV}-2.226696 \mathrm{MeV}=9.812358 \mathrm{MeV}
\end{aligned}
$$

These now provide matrix expressions for intrinsic, latent binding energies of the proton and neutron, contracted down to scalar energy numbers which specify these binding energies and match the empirical data very well. And it is from these, that we learn how to amend the Lagrangian in (1.8) to lay a foundation for consider- ing nuclear binding energies in general.

Contrasting (3.6) and (3.7) with (3.1) and (3.2), we see that in order to match up with the empirical data, the general form of a Lagrangian for the latent binding energy of a nucleon, rather than (1.8), needs to be:

$$
\mathfrak{S}_{\text {binding }}=\frac{1}{2} \operatorname{Tr}\left((2 \pi)^{\frac{3}{2}} F_{\mu \nu} F^{\mu v}-F_{\mu v} \otimes F^{\mu \nu}\right)=\frac{1}{2} \operatorname{Tr}\left((2 \pi)^{\frac{3}{2}} F_{A B} \cdot F_{B D}-F_{A B} \cdot F_{C D}\right)=\frac{1}{2}\left((2 \pi)^{\frac{3}{2}} F_{A B} \cdot F_{B A}-F_{A A} \cdot F_{B B}\right)
$$

Using this, we now start to amend the t'Hooft Lagrangian (9.2) of [1], reproduced below:

$$
\mathfrak{L}=-\frac{1}{4} F_{\mu \nu}^{a} F_{a}^{\mu \nu}-\frac{1}{2} D_{\mu} \phi_{a} D^{\mu} \phi^{a}-\frac{1}{2} \mu^{2} \phi_{a} \phi^{a}-\frac{1}{8} \lambda\left(\phi_{a} \phi^{a}\right)^{2}
$$


First, we apply $\operatorname{Tr} T^{i} T^{j}=\frac{1}{2} \delta^{i j}, F^{\mu v}=T^{i} F_{i}^{\mu \nu}$ and $\Phi=T^{a} \phi_{a}$ to rewrite (3.9) in the Yang-Mills matrix form:

$$
\begin{aligned}
\mathfrak{L} & =-\frac{1}{2} \operatorname{Tr}\left(F_{\mu v} F^{\mu v}\right)-\operatorname{Tr}\left(D_{\mu} \Phi D^{\mu} \Phi\right)-\mu^{2} \operatorname{Tr}(\Phi \Phi)-\frac{1}{2} \lambda(\operatorname{Tr}(\Phi \Phi))^{2} \\
& =-\frac{1}{2} \operatorname{Tr}\left(F_{\mu v A B} F_{B D}^{\mu v}\right)-\operatorname{Tr}\left(\left(D_{\mu} \Phi\right)_{A B}\left(D^{\mu} \Phi\right)_{B D}\right)-\mu^{2} \operatorname{Tr}\left(\Phi_{A B} \Phi_{B D}\right)-\frac{1}{2} \lambda\left(\operatorname{Tr}\left(\Phi_{A B} \Phi_{B D}\right)\right)^{2} \\
& =-\frac{1}{2} F_{\mu v A B} F_{B A}^{\mu v}-\left(D_{\mu} \Phi\right)_{A B}\left(D^{\mu} \Phi\right)_{B A}-\mu^{2} \Phi_{A B} \Phi_{B A}-\frac{1}{2} \lambda\left(\Phi_{A B} \Phi_{B A}\right)^{2}
\end{aligned}
$$

with (9.4) of [1] also written in compacted matrix form:

$$
\left(D_{\mu} \Phi\right)_{A B}=\partial_{\mu} \Phi_{A B}-i\left(\left[G_{\mu}, \Phi\right]\right)_{A B}
$$

Now, we compare (3.10) closely with (3.8), especially comparing $-\frac{1}{2} F_{\mu v A B} F_{B A}^{\mu v}$ in (3.10) with $\frac{1}{2}(2 \pi)^{\frac{3}{2}} F_{A B} \cdot F_{B A}$ in (3.8). Based on this, we reconstruct the t'Hooft Lagrangian so the pure gauge terms specify the latent nuclear binding energies, that is, we choose to make $\frac{1}{2}\left((2 \pi)^{\frac{3}{2}} F_{A B} \cdot F_{B A}-F_{A A} \cdot F_{B B}\right)$ the pure gauge Lagrangian term, because we know from (3.6) and (3.7) that this yields latent binding energies very much in accord with those empirically observed in nuclear physics. Thus, we take (3.10), introduce a factor of $-(2 \pi)^{\frac{3}{2}}$ in front of all the ordinary matrix products, subtract off a term $F_{A A} \cdot F_{B B}$, introduce similarly-contracted terms everywhere else, and so fashion the Lagrangian:

$$
\begin{aligned}
\mathfrak{L}=(2 \pi)^{\frac{3}{2}} & {\left[\frac{1}{2} F_{\mu \nu A B} F_{B A}^{\mu v}+\left(D_{\mu} \Phi\right)_{A B}\left(D^{\mu} \Phi\right)_{B A}+\mu^{2} \Phi_{A B} \Phi_{B A}+\frac{1}{2} \lambda\left(\Phi_{A B} \Phi_{B A}\right)^{2}\right] } \\
& -\frac{1}{2} F_{\mu \nu A A} F_{B B}^{\mu \nu}-\left(D_{\mu} \Phi\right)_{A A}\left(D^{\mu} \Phi\right)_{B B}-\mu^{2} \Phi_{A A} \Phi_{B B}-\frac{1}{2} \lambda\left(\Phi_{A A} \Phi_{B B}\right)^{2}
\end{aligned}
$$

It is readily seen that the pure gauge terms $F_{\mu v} F^{\mu v}$ in the above are identical to (3.8), which means these terms now represent the empirically-observed latent nuclear binding energies. However, in constructing this Lagrangian, we carry the same index structure and $(2 \pi)^{\frac{3}{2}}$ coefficients forward to all remaining terms and thus extend this understanding to the vacuum terms.

The benefit of all of this can be seen by now considering a nucleus with $Z$ protons and $N$ neutrons, which therefore has $A=Z+N$ nucleons. With (3.6) and (3.7), we may write the intrinsic, available, latent binding energy ${ }_{Z}^{A} \mathrm{~B}$ of any such nuclide as:

$$
\begin{aligned}
{ }_{Z}^{A} \mathrm{~B} & =\frac{1}{2} Z \cdot \iiint\left((2 \pi)^{\frac{3}{2}} F_{\mathrm{P} A B} \cdot F_{\mathrm{P} B A}-F_{\mathrm{P} A A} \cdot F_{\mathrm{P} B B}\right) \mathrm{d}^{3} x+\frac{1}{2} N \cdot \iiint\left((2 \pi)^{\frac{3}{2}} F_{\mathrm{N} A B} \cdot F_{\mathrm{N} B A}-F_{\mathrm{N} A A} \cdot F_{\mathrm{N} B B}\right) \mathrm{d}^{3} x \\
& =Z \cdot 7.640679 \mathrm{MeV}+N \cdot 9.812358 \mathrm{MeV}
\end{aligned}
$$

This simply restates the results found in Sections 11 and 12 of [1] in more formal terms. But, it ties formal theoretical expressions based on a Lagrangian

$\mathfrak{L} \propto-\frac{1}{2} \operatorname{Tr}(F \cdot F)$ and an energy $E=-\iiint \mathfrak{L d}^{3} x$ to a very practical formula for deriving real, numeric, empirically-accurate nuclear binding energies. A good example is (1.14) for ${ }_{26}^{56} \mathrm{~B}$, the latent binding energy of ${ }^{56} \mathrm{Fe}$.

On the foregoing basis, we now show how to derive not only the latent, available binding energies (designnated B) via (3.13), but also the observed binding energies (which will be designated throughout as $\mathrm{B}_{0}$ with a "0" subscript) for several basic light nuclides. Specifically, we now lay the foundation for deriving ${ }_{1}^{3} \mathrm{~B}_{0}$ for the ${ }^{3} \mathrm{H}$ triton, ${ }_{2}^{3} \mathrm{~B}_{0}$ for the ${ }^{3} \mathrm{He}$ helion, and most importantly given that it is a fundamental building block of the larger nuclei and many decay process, ${ }_{2}^{4} \mathrm{~B}_{0}$ for the ${ }^{4} \mathrm{He}$ alpha, all extremely closely to the empirical data.

\section{Foundation for Deriving Observed Binding Energies of the 1s Nuclides}

Our goal is to derive the observed, empirical binding energies for all nuclides with $Z \leq 2 ; N \leq 2$ on a totally theoretical basis. We thereby embark on the undertaking set forth at the end of [1], to understand in detail, how collections of Yang-Mills magnetic monopoles - which monopole collections we now understand to be nuclei when the monopoles are protons and neutrons - organize and structure themselves. 
The empirical nuclear weights (masses ${ }_{Z}^{A} M$ ) of the $1 \mathrm{~s}$ nuclides are set forth below in Figure 2 (again, $A=Z+$ $N$ ). Because we wish to do very precise calculations, and because nuclide masses are known much more precisely in $u$ (atomic mass units, $\mathrm{AMU}$ ) than in $\mathrm{MeV}$ due to the "relatively poorly known electronic charge" [11], we shall work in AMU. When helpful for illustration, we shall convert over to $\mathrm{MeV}$ via $1 u=931.494061(21)$ $\mathrm{MeV} / \mathrm{c}^{2}$, but only after a calculation is complete. The data for these nuclides (and the electron mass below) is from [11] and/or [12], and is generally known to ten-digit precision in AMU with experimental errors at the eleventh and twelfth digits. For other nuclides not listed at these sources, we make use of a very helpful online compilation of atomic weights and isotopes at [13]. Vertical columns list isotopes, horizontal rows list isotones, and diagonal lines link isobars of like- $A$. The nuclides with border frames are stable nuclides. The mass of the neutron is $M(n)={ }_{0}^{1} M=1.008664916000 u$ and the mass of the proton is $M(p)={ }_{1}^{1} M=1.007276466812 u$.

The observed binding energies $\mathrm{B}_{0}$ are readily calculated from the above via ${ }_{Z}^{A} \mathrm{~B}_{0}=Z \cdot{ }_{1}^{1} M+N \cdot{ }_{0}^{1} M-{ }_{Z}^{A} M$ using the proton and neutron masses $M(p)={ }_{1}^{1} M$ and $M(n)={ }_{0}^{1} M$, and are summarized in Figure 3 below (again, the observed binding energies will be denoted throughout as $\mathrm{B}_{0}$ with a "0" subscript, while latent, theoretically-available binding energies denoted simply B will omit this subscript).

Now let's get down to business. We already showed in (12.9) of [1] and discussed in the introduction here, that by identifying the mass of the up quark with the deuteron binding energy via the postulate that $m_{u} \equiv \mathrm{B}_{0}\left({ }^{2} \mathrm{H}\right)=$ $2.224566 \mathrm{MeV}$, we not only can establish very precise masses for the up and down quarks but also can explain the confluence of confinement and fission and fusion at ${ }^{56} \mathrm{Fe}$ in a very profound way, wherein $99.8429093 \%$ of the available binding energy goes into binding the ${ }^{56} \mathrm{Fe}$ nucleus and only the remaining $0.1570907 \%$ is unused for nucleon binding and so instead confines quarks. And, we extrapolated this to the thesis to be further confirmed here, that nucleons in general are resonant cavities fusing at energies reflective of their current quark masses.

So we now write this postulate identifying (defining) the up quark mass $m_{u}$ with the observed deuteron binding energy ${ }_{1}^{2} \mathrm{~B}_{0}$, in notations to be employed here, in AMU, as:

$$
m_{u} \equiv{ }_{1}^{2} \mathrm{~B}_{0}=0.002388170100 u .
$$

In AMU, the electron mass, which we shall also need, is:

$$
m_{e}=0.000548579909 u .
$$

We then use (1.11) (see also (12.10) of [1]) with (4.1) and (4.2) to obtain the down quark mass:

$$
m_{d}=(2 \pi)^{\frac{3}{2}} m_{e} / 3+m_{u}=0.005268143299 u .
$$

It will also be helpful in the discussion following to use:

$$
\sqrt{m_{u} m_{d}}=0.003547001876 u
$$

see, e.g., (1.12) and (1.13) in which this first arises.

We then use the foregoing in (1.12) and (1.13) to calculate the latent, available binding energy of the proton and neutron, designated B without the " 0 " subscript:

$$
\begin{aligned}
\mathrm{B}(p) & ={ }_{1}^{1} \mathrm{~B}=2 m_{u}+m_{d} \\
& -\left(m_{d}+4 \sqrt{m_{u} m_{d}}+4 m_{u}\right) /(2 \pi)^{\frac{3}{2}} \\
& =0.008202607332 u
\end{aligned}
$$

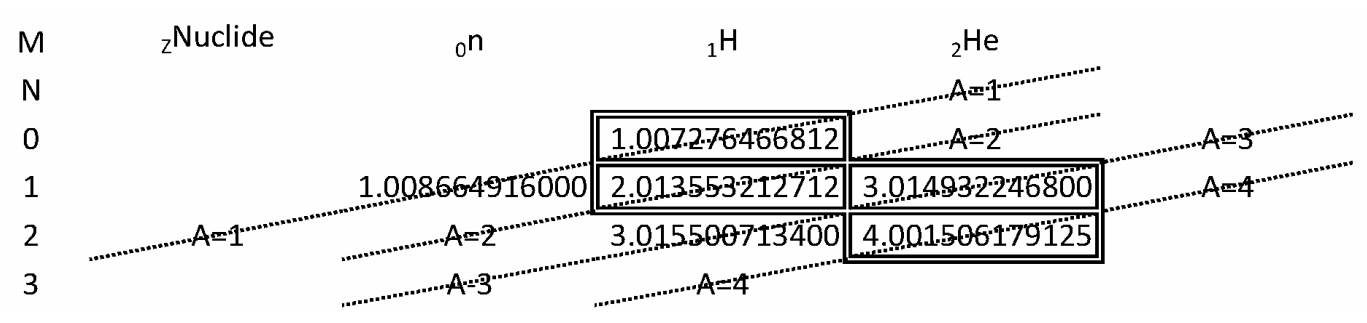

Figure 2. Empirical nuclear weights $\left({ }_{z}^{A} M\right)$ of 1s nuclides (AMU).

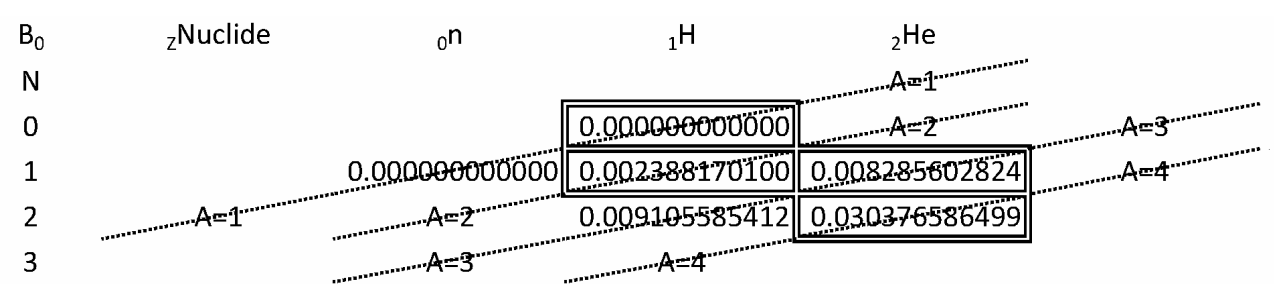

Figure 3. Empirical binding energies $\left({ }_{z}^{A} B_{0}\right)$ of 1s nuclides (AMU). 


$$
\begin{aligned}
\mathrm{B}(n) & ={ }_{0}^{1} \mathrm{~B}=2 m_{d}+m_{u} \\
& -\left(m_{u}+4 \sqrt{m_{u} m_{d}}+4 m_{d}\right) /(2 \pi)^{\frac{3}{2}} \\
& =0.010534000622 u
\end{aligned}
$$

Via (3.13), (4.5) and (4.6) may then be used to calculate generally, the latent, available binding energy:

$$
\begin{aligned}
{ }_{Z}^{A} \mathrm{~B} & =Z \cdot\left(2 m_{u}+m_{d}-\frac{m_{d}+4 \sqrt{m_{u} m_{d}}+4 m_{u}}{(2 \pi)^{\frac{3}{2}}}\right) \\
& +N \cdot\left(2 m_{d}+m_{u}-\frac{m_{u}+4 \sqrt{m_{u} m_{d}}+4 m_{d}}{(2 \pi)^{\frac{3}{2}}}\right) \\
& =Z \cdot 0.008202607332 u+N \cdot 0.010534000622 u
\end{aligned}
$$

for any nuclide of given $Z, N$. For the nuclides in Figures 2 and 3, this theoretically-available, latent binding energy $\mathrm{B}$, is predicted to be: see Figure 4.

Taking the ratio of the empirical values in Figure 3 over the theoretical values in Figure 4 and expressing these as percentages then yields: see Figure 5.

So we see, for example, that the ${ }^{4} \mathrm{He}$ alpha nucleus uses about $81.06 \%$ of its total available latent binding energy to bind itself together, with the remaining $18.94 \%$ retained to confine the quarks inside each nucleon. The deuteron releases about $12.74 \%$ of it latent binding energy for nuclear binding, while the isobars with $A=3$ release about $31 \%$ of this latent energy for nuclear binding with the balance reserved for quark confinement. The free proton and neutron, of course, retain $100 \%$ of this latent energy to bind their quarks and release nothing. So one may think of the latent binding energy as an energy that "see-saws" between confining quarks and binding together nucleons into nuclides, with the exact percentage of latent energy reserved for quark confinement ver- sus released for nuclear binding dependent on the particular nuclide in question.

As a point of comparison, we return to ${ }^{56} \mathrm{Fe}$ which has the highest percentage of used-to-available binding energy of any nuclide. Its nuclear weight

${ }_{26}^{56} M=55.92067442 u$ (cf. Figure 2), its empirical, observed binding energy ${ }_{26}^{56} \mathrm{~B}_{0}=0.52846119 u$ (cf. Figure 3), its latent binding energy ${ }_{26}^{56} \mathrm{~B}=0.52928781 u$ (cf Figure 4), and its used-to-available percentage ${ }_{26}^{56} \mathrm{~B}_{0} /{ }_{26}^{56} \mathrm{~B}(\%)=99.843825 \%$ (cf. Figure 5). No nuclide has a higher such percentage than ${ }^{56} \mathrm{Fe}$. While ${ }^{62} \mathrm{Ni}$ has a larger empirical binding energy per-nucleon, its used-toavailable percentage is lower, because the calculation in (4.7) literally and figuratively weights the neutrons more heavily than the protons by a ratio of:

$$
\begin{aligned}
\frac{\mathrm{B}(n)}{\mathrm{B}(p)} & =\frac{{ }_{0}^{1} \mathrm{~B}}{{ }_{1}^{1} \mathrm{~B}}=\frac{0.010534000622 u}{0.008202607332 u} \\
& =1.284225880325
\end{aligned}
$$

The above ratio explains the long-observed phenomenon why heavier nuclides tend to have a greater number of neutrons than protons: For heavier nuclides, because the neutrons carry an energy available for binding which is about $28.42 \%$ larger than that of the proton, neutrons will in general find it easier to bind into a heavy nucleus by a factor of $28.42 \%$. Simply put: neutrons bring more available binding energy to the table than protons and so are more welcome at the table. The nuclides running from ${ }^{31} \mathrm{Ga}$ to ${ }^{48} \mathrm{Cd}$ tend to have stable isotopes with neutron-to-proton number ratios $(N / Z)$ roughly in the range of (4.8). Additionally, and likely for the same reason, this is the range in which, beginning with ${ }^{41} \mathrm{Nb}$ and ${ }^{42} \mathrm{Mo}$, and as the $\mathrm{N} / \mathrm{Z}$ ratio grows even larger than (4.8), one begins to see nuclides which become theoretically unstable with regard to spontaneous fission.

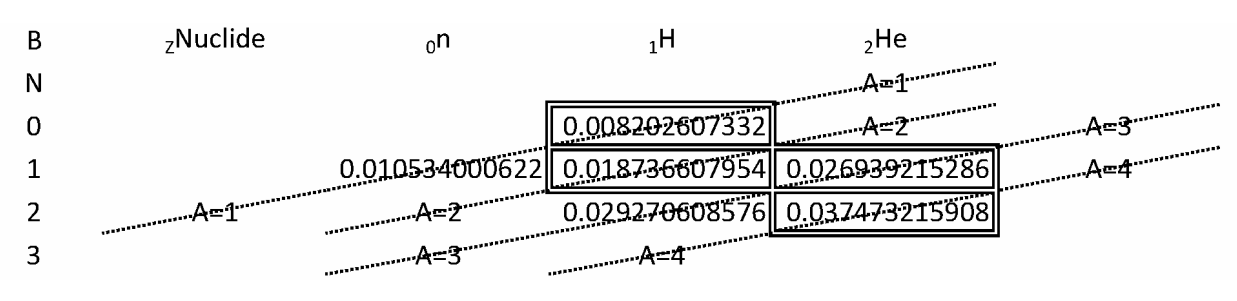

Figure 4. Theoretically available binding energies $\left({ }_{z}^{A} B\right)$ of 1 s nuclides (AMU).

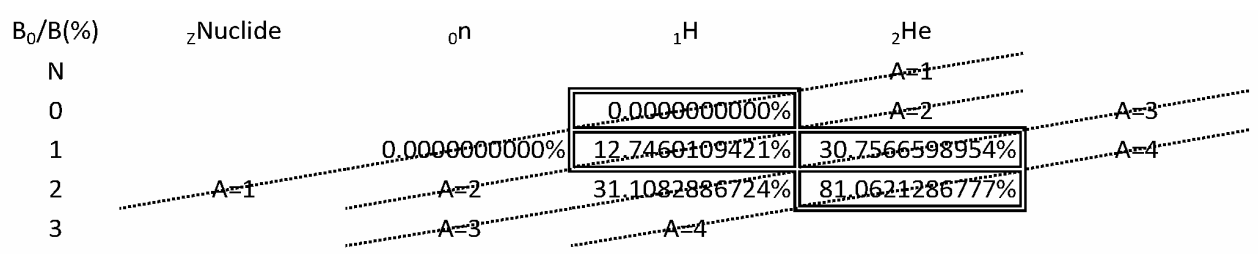

Figure 5. Used-to-available binding energies $\left({ }_{z}^{A} \mathbf{B}_{0} /{ }_{Z}^{A} \mathbf{B}(\%)\right)$ of 1 s nuclides (\%). 
Next, we subtract Figure 3 from Figure 4, to obtain the unused $(U)$ binding energy ${ }_{Z}^{A} U={ }_{Z}^{A} B-{ }_{Z}^{A} B_{0}$ for each nuclide. These unused binding energies represent the amount of the latent binding energies reserved for and channeled into intra-nucleon quark confinement, rather than released and used for inter-nucleon binding. Of course, for the proton and neutron, all of this energy is unused; it is fully reserved and channeled into confining the quarks. These unused, reserved-for-confinement energies are: see Figure 6.

Finally, to lay the groundwork for predicting the observed binding energies $\mathrm{B}_{0}$ in Figure 3, let us refer to (3.6) and (3.7), remove the trace, and specify two $(3 \times 3)$ $\times(3 \times 3)$ outer product matrices, one for the proton, $E_{\mathrm{P} A B C D}$, and one for the neutron, $E_{\mathrm{N} A B C D}$, according to:

$$
\begin{aligned}
& (2 \pi)^{\frac{3}{2}} E_{\mathrm{P} A B C D}=\frac{1}{2}(2 \pi)^{\frac{3}{2}} \iiint F_{\mathrm{P} A B} \cdot F_{\mathrm{P} C D} \mathrm{~d}^{3} x \\
& =\left(\begin{array}{ccc}
\sqrt{m_{d}} & 0 & 0 \\
0 & \sqrt{m_{u}} & 0 \\
0 & 0 & \sqrt{m_{u}}
\end{array}\right) \otimes\left(\begin{array}{ccc}
\sqrt{m_{d}} & 0 & 0 \\
0 & \sqrt{m_{u}} & 0 \\
0 & 0 & \sqrt{m_{u}}
\end{array}\right) \\
& (2 \pi)^{\frac{3}{2}} E_{\mathrm{N} A B C D}=\frac{1}{2}(2 \pi)^{\frac{3}{2}} \iiint F_{\mathrm{N} A B} \cdot F_{\mathrm{N} C D} \mathrm{~d}^{3} x \\
& =\left[\left(\begin{array}{ccc}
\sqrt{m_{u}} & 0 & 0 \\
0 & \sqrt{m_{d}} & 0 \\
0 & 0 & \sqrt{m_{d}}
\end{array}\right) \otimes\left(\begin{array}{ccc}
\sqrt{m_{u}} & 0 & 0 \\
0 & \sqrt{m_{d}} & 0 \\
0 & 0 & \sqrt{m_{d}}
\end{array}\right)\right]
\end{aligned}
$$

From the above, one can readily obtain the eighteen non-zero diagonal outer product components (nine for the proton and nine for the neutron), with $E_{\mathrm{P} A B C D}=E_{\mathrm{N} A B C D}$ $=0$ otherwise:

$$
\begin{aligned}
E_{\mathrm{N} 1111} & =E_{\mathrm{P} 2222}=E_{\mathrm{P} 3333}=E_{\mathrm{P} 2233} \\
& =E_{\mathrm{P} 3322}=m_{u} /(2 \pi)^{\frac{3}{2}} \\
E_{\mathrm{P} 1111} & =E_{\mathrm{N} 2222}=E_{\mathrm{N} 3333}=E_{\mathrm{N} 2233} \\
& =E_{\mathrm{N} 3322}=m_{d} /(2 \pi)^{\frac{3}{2}} \\
E_{\mathrm{P} 1122} & =E_{\mathrm{P} 1133}=E_{\mathrm{P} 2211}=E_{\mathrm{P} 3311} \\
& =E_{\mathrm{N} 1122}=E_{\mathrm{N} 1133}=E_{\mathrm{N} 2211} \\
& =E_{\mathrm{N} 3311}=\sqrt{m_{u} m_{d}} /(2 \pi)^{\frac{3}{2}}
\end{aligned}
$$

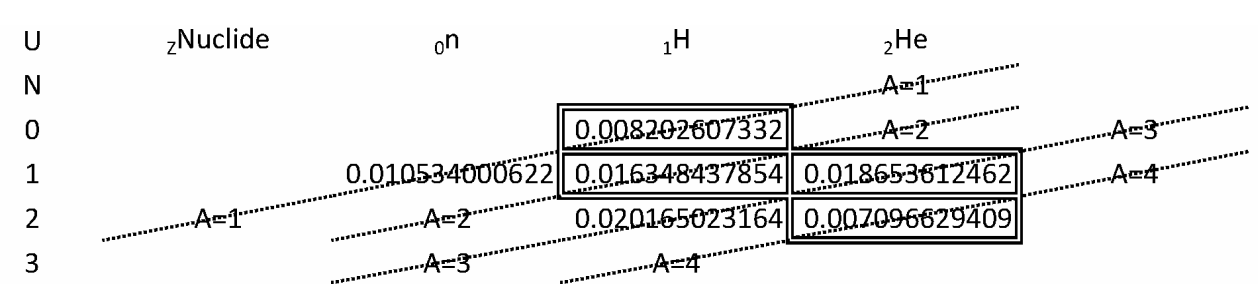

Figure 6. Unused latent binding energies $\left({ }_{Z}^{A} U\right)$ of 1 s nuclides (AMU). ing block of nuclear physics. theoretical reasons why this may make sense. dicted to be (4.7) for ${ }_{2}^{4} \mathrm{~B}$, less $2 \sqrt{m_{u} m_{d}}$, that is:

$$
\begin{aligned}
{ }_{2}^{4} \mathrm{~B}_{0 \text { Predicted }} & =2 \cdot\left(2 m_{u}+m_{d}-\frac{m_{d}+4 \sqrt{m_{u} m_{d}}+4 m_{u}}{(2 \pi)^{\frac{3}{2}}}\right) \\
& +2 \cdot\left(2 m_{d}+m_{u}-\frac{m_{u}+4 \sqrt{m_{u} m_{d}}+4 m_{d}}{(2 \pi)^{\frac{3}{2}}}\right) \\
& -2 \sqrt{m_{u} m_{d}}=0.030379212155 u
\end{aligned}
$$

This is why (4.1), (4.3) and (4.4) will be of interest in the development following. With the "toolkit" (4.9) to (4.11) we now have all ingredients needed to closely deduce the empirical binding energies in Figure 3 on totally theoretical grounds. We start with the alpha, ${ }^{4} \mathrm{He}$.

\section{Prediction of the Alpha Nuclide Binding Energy to 3 Parts in One Million, and How Binding Energies Are Yang-Mills Tensor Components}

The alpha particle is the ${ }^{4} \mathrm{He}$ nucleus. It is highly stable, with fully saturated $1 \mathrm{~s}$ shells for protons and neutrons, and is central to many aspects of nuclear physics including the decay of nuclides into more stable states via so-called alpha decay. In this way, it is a bedrock build-

The unused binding energy in Figure 6 for the alpha is ${ }_{2}^{4} U=0.007096629409 u$. Looking over the toolkit (4.11), we see $2 \sqrt{m_{u} m_{d}}=0.007094003752 u$, so ${ }_{2}^{4} U$ is very close to being twice the value of $\sqrt{m_{u} m_{d}}$ in (4.4). In fact, these energies are equal to about 2.26 parts per million! Might this be an indication that the alpha uses all its latent binding energy less $2 \sqrt{m_{u} m_{d}}$ for nuclear binding, with the $2 \sqrt{m_{u} m_{d}}$ balance reserved on the other side of the "see saw" to confine quarks within each of its four nucleons? First, let's look at the numbers, then examine

If in fact this numerical coincidence is not just a coincidence but has real physical meaning, this would mean the empirical binding energy ${ }_{2}^{4} \mathrm{~B}_{0}$ of the alpha is pre-

where we calculate using $m_{u}, m_{d}$ from (4.1), (4.3), and 
$\sqrt{m_{u} m_{d}}$ from (4.4). In contrast, the empirical ${ }_{2}^{4} \mathrm{~B}_{0}=$ $0.030376586499 u$ in Figure 3. The difference:

$$
\begin{aligned}
{ }_{2}^{4} \mathrm{~B}_{0 \text { Predicted }}-{ }_{2}^{4} \mathrm{~B}_{0} & =0.030379212155 u \\
& -0.030376586499 u \\
& =0.000002625656 u
\end{aligned}
$$

is extremely small, with these two values, as noted just above for the reserved energy, differing from one another by less than 3 parts in 1 million AMU! So, let us regard (5.1) to be a correct prediction of the alpha binding energy to 3 parts per million. Now, let's discuss the theoretical reasons why this makes sense.

In [1], a key postulate was to identify the mass of the down quark with the deuteron binding energy, see (4.1) here in which we again reviewed that identification. Beyond the numerical concurrence, a theoretical explanation is that in some fashion the nucleons are resonant cavities, so the energies they release (or reserve) during fusion will be very closely tied to the masses/wavelengths of the contents of these cavities. But, of course, these "cavities" contain up quarks and down quarks, and their masses are given in (4.1) and (4.3) together with the $\sqrt{m_{u} m_{d}}$ construct in (4.4), and so these will specify preferred "harmonics" to determine the precise energies which these cavities resonantly release for nuclear binding, or hold in reserve for quark confinement.

We also see that components of the outer products

$$
(2 \pi)^{\frac{3}{2}} E_{A B C D}=\frac{1}{2}(2 \pi)^{\frac{3}{2}} \iiint F_{A B} \cdot F_{C D} \mathrm{~d}^{3} x \text { in (4.9) and (4.10) }
$$

take on one of three non-zero values: $m_{u}, m_{d}$, or $\sqrt{m_{u} m_{d}}$, see (4.11). So, in trying to make a theoretical fit to empirical binding data we require that empirical binding energies be calculated only from these outer products $E_{A B C D}=\frac{1}{2} \iiint F_{A B} \cdot F_{C D} \mathrm{~d}^{3} x$ (4.9), (4.10) using only some combination of 1) the components of these outer products and 2) index contractions of these outer products. So the ingredients we shall use to do this numerical fitting will be restricted to 1) the latent nuclide binding energies calculated from $(4.7) ; 2$ ) the three energies $m_{u}, m_{d}$, $\sqrt{m_{u} m_{d}}$ of (4.11) and quantized multiples thereof; and 3) any of the foregoing with a $(2 \pi)^{\frac{3}{2}}$ coefficient or divisor, as suitable; we also permit 4) the rest mass of the electron $m_{e}$ which is related to the up and down masses via (1.11). The method of this fitting is trial and error, at least for now, and involves essentially poring over the empirical nuclear binding energy data and seeing if it can be arrived at closely using only the foregoing ingredients.

For the alpha, (5.1) meets all these criteria. In fact, rewritten with (3.6), (3.7) and (4.9) through (4.11), we find (5.1) can be expressed entirely in terms of the outer product $E_{A B C D}=\frac{1}{2} \iiint F_{A B} \cdot F_{C D} \mathrm{~d}^{3} x$ as just discussed, as:

$$
\begin{aligned}
{ }_{2}^{4} \mathrm{~B}_{0 \text { Predicted }} & =2 \cdot\left((2 \pi)^{\frac{3}{2}} E_{\mathrm{P} A B B A}-E_{\mathrm{P} A A B B}\right) \\
& +2 \cdot\left((2 \pi)^{\frac{3}{2}} E_{\mathrm{N} A B B A}-E_{\mathrm{N} A A B B}\right) \\
& -(2 \pi)^{\frac{3}{2}}\left(E_{\mathrm{P} 1122}+E_{\mathrm{N} 1122}\right) \\
= & 2 \cdot\left(2 m_{u}+m_{d}-\frac{m_{d}+4 \sqrt{m_{u} m_{d}}+4 m_{u}}{(2 \pi)^{\frac{3}{2}}}\right) \\
& +2 \cdot\left(2 m_{d}+m_{u}-\frac{m_{u}+4 \sqrt{m_{u} m_{d}}+4 m_{d}}{(2 \pi)^{\frac{3}{2}}}\right) \\
& -2 \sqrt{m_{u} m_{d}}
\end{aligned}
$$

This totally theoretical Yang-Mills tensor expression yields the alpha binding energy to 2.26 parts per million.

In this form, (5.3) tells us that the alpha binding energy is actually the 1122 component of a $(3 \times 3) \times(3 \times 3)$ outer product $E_{A B C D}$, in linear combination with traces of $E_{A B C D}$. That is, this binding energy is a component of a Yang-Mills tensor!

This is reminiscent, for example, of the Maxwell Tensor $-4 \pi T^{\mu v}=F^{\mu \alpha} F_{\alpha}^{v}-\frac{1}{4} \eta^{\mu v} F^{\alpha \beta} F_{\alpha \beta}$, which provides a suitable analogy. The on-diagonal components of the Maxwell tensor contain both a component term and a trace term just like (5.3). For example, for the 00 term $-4 \pi T^{00}=F^{0 \alpha} F_{\alpha}^{0}-\frac{1}{4} F^{\alpha \beta} F_{\alpha \beta}$, we analogize $F^{0 \alpha} F_{\alpha}^{0}$ to the $E_{1122}$ and $F^{\alpha \beta} F_{\alpha \beta}$ to the $(2 \pi)^{\frac{3}{2}} E_{A B B A}-E_{A A B B}$ in (5.3). The off-diagonal components of the Maxwell tensor, however, do not include a trace term. For example, for the 01 term in Maxwell, if we consider $-4 \pi T^{01}=$ $F^{0 \alpha} F_{\alpha}^{1}-\eta^{01} \frac{1}{4} F^{\alpha \beta} F_{\alpha \beta}=F^{0 \alpha} F_{\alpha}^{1}+0$, the Minkowski metric $\eta^{\mu v}$ filters out the trace. This latter, off-diagonal analogy allows us to represent (4.1) for the deuteron as a tensor component without a trace term, for example, as (see (4.11)):

$$
{ }_{1}^{2} \mathrm{~B}_{0 \text { Predicted }}=m_{u}=(2 \pi)^{\frac{3}{2}} E_{\mathrm{N} 1111}+0 .
$$

So we now start to think about individual observed nuclear binding energies as components of a fourth rank Yang Mills tensor of which (5.3) and (5.4) are merely two samples. Thus, as we proceed to examine many different nuclides, we will want to see what patterns may be discerned for how each nuclide fits into this tensor. 
Physically, the alpha particle contains two protons and two neutrons, in terms of quarks, six up quarks and six down quarks. It is seen that the up quarks enter (5.3) in a completely symmetric fashion relative to the down quarks, i.e., that (5.3) is invariant under the interchange $m_{u} \leftrightarrow m_{d}$. The factor of 2 in front of $\sqrt{m_{u} m_{d}}$ of course means that two components of the outer product are also involved. So we have preliminarily associated $2 \sqrt{m_{u} m_{d}}=$ $E_{\mathrm{P} 1122}+E_{\mathrm{N} 1122}$ so that the neutron pair and the proton pair each contribute $1 \sqrt{m_{u} m_{d}}$ to (5.3), and (5.3) thereby remains absolutely symmetric not only under $u \leftrightarrow d$, but also under $p \leftrightarrow n$ interchange.

We do note that there is some flexibility in these assignments of energy numbers to tensor components, because each of $m_{u}, m_{d}, \sqrt{m_{u} m_{d}}$ in the (4.11) toolkit is associated with several different components of the outer product. So the choice of $E_{1122}$ in (5.3) (while requiring $p \leftrightarrow n$ symmetry) and of $E_{\mathrm{N} 1111}$ in (5.4) is flexible versus the other available possibilities in (4.11), and should be revisited once we study other nuclides not yet considered and seek to understand the more general Yang-Mills tensor structure of which the individual nuclide binding energies are components.

One other physical observation is also very noteworthy, and to facilitate this discussion we include the wellknown "per-nucleon" binding graph as Figure 1 above. One perplexing mystery of nuclear physics is why there is such a large "chasm" between binding energies for the ${ }^{2} \mathrm{H},{ }^{3} \mathrm{H}$ and ${ }^{3} \mathrm{He}$ nuclides, and the biding energy of the ${ }^{4} \mathrm{He}$ nuclide which we have now predicted to within parts per million. Contrasting (5.3) for ${ }^{4} \mathrm{He}$ with (5.4) for ${ }^{2} \mathrm{H}$, we see that for the latter deuteron, we "start at the bottom" with ${ }_{1}^{1} \mathrm{~B}_{0}=0$ for ${ }^{1} \mathrm{H}$ (the free proton), and then "add" ${ }_{1}^{2} \mathrm{~B}_{0}=0+m_{u}$ worth of energy to bind the proton and the neutron together into ${ }^{2} \mathrm{H}$. Conversely, for the alpha we "start at the top" with the total latent binding energy ${ }_{2}^{4} \mathrm{~B}=0.037473215908 u$, and then subtract off

$2 \sqrt{m_{u} m_{d}}$, to match the empirical data with

${ }_{2}^{4} \mathrm{~B}_{0}=0.037473215908 u-2 \sqrt{m_{u} m_{d}}$. But as we learned in Section 12 of [1] and have reiterated here, any time we do not use some of the latent energy for nuclear binding, that unused energy remains behind in reserve to confine the quarks in a type of nuclear see-saw.

So what we learn is that for the alpha particle, a total of $2 \sqrt{m_{u} m_{d}}=0.007094004 u$ is held in reserve to confine the quarks, while the majority balance is released to bind the nucleons to one another. In contrast, for the deuteron, a total of $m_{u} \equiv{ }_{1}^{2} \mathrm{~B}_{0}=0.002388170100 u$ is released for inter-nucleon binding while the majority balance is held in reserve to confine the quarks.

Now to the point: for some nuclides (e.g. the deuteron) the question is: how much energy is released from quark confinement to bind nucleons? This is a "bottom to top" nuclide. For other nuclides (e.g., the alpha) the question is: how much energy is reserved out of the theoretical maximum available, to confine quarks. This is a "top to bottom" nuclide. For top to bottom nuclides, there is a scalar trace in the Yang-Mills tensors. For bottom to top nuclides there is not. Using the Maxwell tensor analogy, one may suppose that somewhere there is a Kronecker delta $\delta_{B}^{A}$ and/or $\delta_{C D}^{A B}$ which filters out the trace from "off-diagonal" terms and leaves the trace intact for "ondiagonal" terms. In this way, the "bottom to top" nuclides are "off-diagonal" tensor components and the "top to bottom" nuclides are "on diagonal" components. In either case, however, the "resonance" for nuclear binding is established by the components of the $E_{\mathrm{N} A B C D}$, which are $m_{u}, m_{d}, \sqrt{m_{u} m_{d}}$ in some combination and/or integer multiple. And, as regards Figure 1 above, the chasm between the lighter nuclides and ${ }^{4} \mathrm{He}$ is explained on the basis that each of ${ }^{2} \mathrm{H},{ }^{3} \mathrm{H}$ and ${ }^{3} \mathrm{He}$ are "bottom to top" "off-diagonal" nuclides, while ${ }^{4} \mathrm{He}$, which happens to fill the 1s shells, is the lightest "top to bottom" "on-diagonal" nuclide. ${ }^{2} \mathrm{H},{ }^{3} \mathrm{H}$ and ${ }^{3} \mathrm{He}$ start at the bottom of the nuclear see-saw and move up; ${ }^{4} \mathrm{He}$ starts at the top of the see-saw and moves down.

To amplify this point, in Figure 7 below we peek ahead at some heavier nuclides, namely, ${ }_{3} \mathrm{Li}$ and ${ }_{4} \mathrm{Be}$. Using a nuclear shell model similar to that used for electron structure, all nucleons in the ${ }^{4} \mathrm{He}$ alpha are in $1 \mathrm{~s}$ shells. The two protons are spin up and down each with $1 \mathrm{~s}$, as are the two neutrons. As soon as we add one more nucleon, by Exclusion, we must jump up to the 2 s shell, which admits four more nucleons and can reach up to ${ }_{4}^{8} \mathrm{Be}$ before we must make an incursion into the $2 \mathrm{p}$ shell.

We note immediately from the above - which has been noticed by others before - that the binding energy ${ }_{4}^{8} \mathrm{~B}_{0}=0.060654752 u$ of ${ }^{8} \mathrm{Be}$ is almost twice as large as that of the alpha particle, to just under one part in ten thousand AMU. Specifically:

$$
\begin{aligned}
2 \cdot{ }_{2}^{4} \mathrm{~B}_{0}-{ }_{4}^{8} \mathrm{~B}_{0} & =2 \times 0.030376586499 u \\
& -0.060654752 u \\
& =0.000098421 u
\end{aligned}
$$

This is part of why ${ }^{8} \mathrm{Be}$ is unstable and invariably decays almost immediately into two alpha particles $\left({ }^{9} \mathrm{Be}\right.$ is the stable $\mathrm{Be}$ isotope). But of particular interest here, is to subtract off the alpha ${ }_{2}^{4} \mathrm{~B}_{0}=0.030376586499 u$ from each of the $\mathrm{Li}$ and $\mathrm{Be}$ isotopes, and compare them side by side with the non-zero binding energies from $\mathrm{H}$ and He. The result of this exercise is in Figure 8 below.

Equation (5.5) is represented above by the fact that ${ }_{4}^{8} \mathrm{~B}_{0}-{ }_{2}^{4} \mathrm{~B}_{0} \cong{ }_{2}^{4} \mathrm{~B}$. The table on the left is a "1s square" and the table on the right is a " 2 s square." But they are both "s-squares." What is of interest is that the remaining three nuclides in the $2 \mathrm{~s}$ square are not dissimilar in pat- 
tern from the other three nuclides in the 1s square. This means that three of the four nuclides in the $2 \mathrm{~s}$ square start "at the bottom" "off-diagonal" just as in $1 \mathrm{~s}$, and the fourth, ${ }^{8} \mathrm{Be}$, starts "on diagonal" "at the top." But, in the $2 \mathrm{~s}$ square, the "bottom" is the alpha particle's ${ }_{2}^{4} \mathrm{~B}_{0}=$ $0.030376586499 u$. So the filled $1 \mathrm{~s}$ shell provides a "platform" below the $2 \mathrm{~s}$ shell; a non-zero minimum energy underpinning binding in the $2 \mathrm{~s}$ square. And it appears at least from the $1 \mathrm{~s}$ and $2 \mathrm{~s}$ examples that nuclides with full shells are "diagonal" tensor components and all others are off diagonal. The see-saw for $2 \mathrm{~s}$ is elevated so its bottom is at the top of the 1s see-saw.

It is also important to note that as we consider much heavier nuclides - and ${ }^{56} \mathrm{Fe}$ is the best example-even more of the energy that binds quarks together is released from all the nucleons. For ${ }^{56} \mathrm{Fe}$, calculating from the discussion prior to (4.8), the unused $U$ binding energy contributed by all 56 nucleons totals only $0.00082662 u$. But in Figure 6 we saw that $0.00709663 u$ of the ${ }^{4} \mathrm{He}$ binding energy is unused. Much of this, therefore, is clearly used by the time one arrives at ${ }^{56} \mathrm{Fe}$. So, almost all the binding energy that is reserved for quark confinement for lighter nuclides becomes released to bind together heavier nuclides, with peak utilization at ${ }^{56} \mathrm{Fe}$. That is, by the time an ${ }^{56} \mathrm{Fe}$ nuclide has been fused together, much of the binding energy previously reserved in the $1 \mathrm{~s}$ and $2 \mathrm{~s}$ shells to confine quarks has been released, and this contributes to overall binding for the heavier nuclides. One may thus think of the unused binding energy in lighter nuclides as a "reservoir" of energy that will be called upon for binding together heavier nuclides. For nuclides heavier than ${ }^{56} \mathrm{Fe}$, the used-to-available percentage, cf. Figure 1, tacks downwards again, and more energy is channeled back into quark confinement and less into nuclear binding. So while quark confinement is "bent" to the limit at ${ }^{56} \mathrm{Fe}$, with almost all latent binding energies see-sawed into nucleon binding rather than quark confinement, quark confinement can never be "broken."

Finally, before turning to ${ }^{3} \mathrm{He}$ in the next section, let us comment briefly on experimental errors. The prediction of ${ }_{2}^{4} \mathrm{~B}_{0 \text { Predicted }}=0.030379212155 u$ for the alpha in (5.1), in contrast to ${ }_{2}^{4} \mathrm{~B}_{0}=0.030376586499 u$ from the empirical data, is an exact match in AMU through the fifth decimal place, but is still not within experimental errors. Specifically, the alpha mass listed in [12] and shown in Figure 2 is $4.001506179125(62) u$, which is accurate to ten decimal places in AMU. Similarly, the proton mass $1.007276466812(90) u$ and the neutron mass

$1.00866491600(43) u$ used to calculate ${ }_{2}^{4} \mathrm{~B}_{0}$ are accurate to ten and nine decimal places respectively in AMU. So the match between ${ }_{2}^{4} \mathrm{~B}_{0 \text { Predicted }}$ and the empirical ${ }_{2}^{4} \mathrm{~B}_{0}$ to under 3 parts per million is still not within the experimental errors beyond five decimal places, because this energy is known to at least nine decimal places in AMU. Consequently, (5.1) must be regarded as a very close, but still approximate relationship for the observed alpha binding energy. Additionally, because (5.1) is based on (4.1), wherein the mass of the up quark is identified with $m_{u} \equiv{ }_{1}^{2} \mathrm{~B}_{0}=0.002388170100 u$ which is the deuteron binding energy, the question must be considered whether this identification (4.1), while very close, is also still approximate.

Specifically, it is possible to make (5.1) for the alpha into an exact relationship, within experimental errors, if we reduce the up quark mass by exactly $\varepsilon=$ $0.000000351251415 u$ (in the seventh decimal place), such that:

$$
\begin{aligned}
m_{u} & =0.002387818849 u \cong{ }_{1}^{2} \mathrm{~B}_{0} \\
& =0.002388170100 u
\end{aligned}
$$

That is, we can make (5.1) for the alpha into an exact

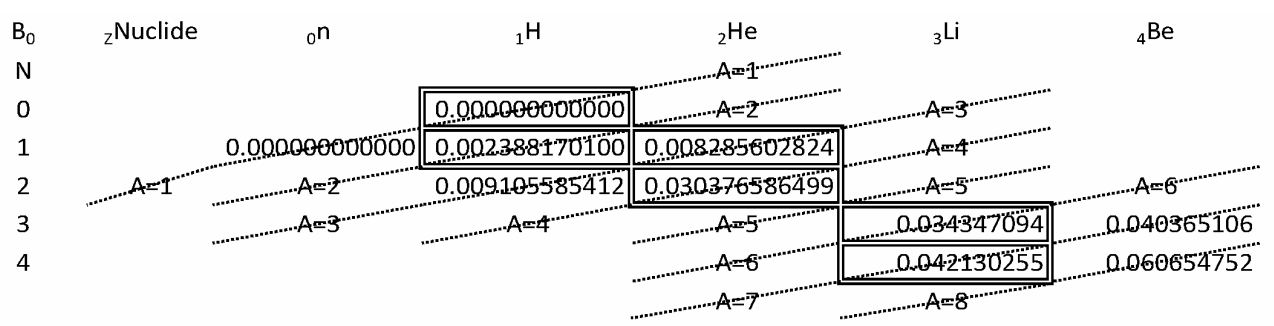

Figure 7. Empirical binding energies $\left({ }_{z}^{A} B_{0}\right)$ of selected 1s and 2s nuclides (AMU).
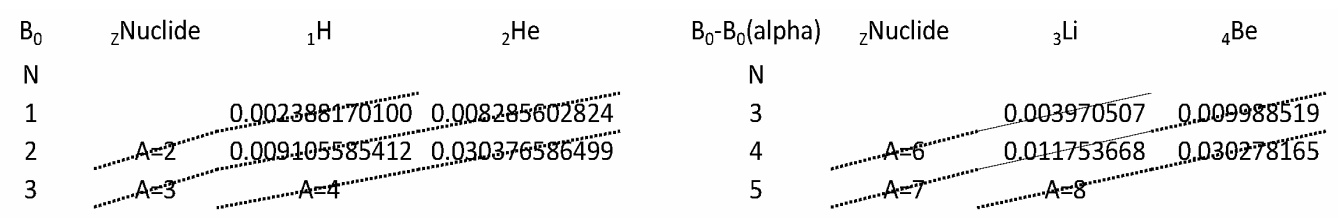

Figure 8. Comparison of Alpha-subtracted 2s binding energies, with 1s binding energies (AMU). 
relationship if we make (4.1) for the up quark into an approximate relationship, or vice versa, but not both. So, should we do this?

A further clue is provided by (5.5), whereby the $\mathrm{em}$ pirical ${ }_{4}^{8} \mathrm{~B}_{0} /{ }_{2}^{4} \mathrm{~B}_{0} \cong 2$ is a close, but still approximate relationship. This close but not exact ratio is not a comparison between a theoretical prediction and empirical observation; it is a comparison between two empirical data points. So this seems to suggest, as one adds more nucleons to a system and makes empirical predictions such as (5.1) based on the up and down quark masses, that higher order corrections (at the sixth decimal place in AMU for alpha and the fifth decimal place in AMU for ${ }_{4}^{8} \mathrm{~B}_{0}$ ) will still be needed. So because two-body systems such as the deuteron can generally be modeled nearly-exactly, and because a deuteron will suffer less from "large $A=Z+N$ corrections" than any other nuclide, it makes sense absent evidence to the contrary to regard (4.1) identifying the up quark mass with the deuteron binding energy to be an exact relationship, and to regard (5.1) for the alpha to be an approximate relationship that still requires some tiny correction in the sixth decimal place. Similarly, as we develop other relationships which, in light of experimental errors, are also close but still approximate, we shall take the view that these relationships too, especially given (5.5), will require higher order corrections. Thus, for the moment, we leave (4.1) intact as an exact relationship.

In section 10 , however, we shall show why (4.1) is actually not an exact relationship but is only approximate to about 8 parts per ten million AMU. But this will be due not to the closeness of the predicted-versus-observed energies for the alpha particle, but due to our being able to develop a theoretical expression for the difference $M(n)-M(p)$ between the observed masses of the free neutron and the free proton to better than one part per million AMU.

\section{Prediction of the Helion Nuclide Binding Energy to 4 Parts in 100,000}

Now, we turn to the ${ }_{2}^{3} \mathrm{He}$ nucleus, also referred to as the helion. In contrast with the alpha and the deuteron already examined which are integer-spin bosons, this nucleon is a half-integer spin fermion. Knowing as pointed out after (5.4) that we will "start at the bottom" of the see-saw for this nuclide, and knowing that our toolkit for constructing binding energy predictions is $m_{u}, m_{d}, \sqrt{m_{u} m_{d}}$, it turns out after some trial and error exercises strictly with these energies that we can make a fairly close prediction by setting:

$$
\begin{aligned}
\mathrm{B}_{0}\left({ }^{3} \mathrm{He}\right)_{\text {Predicted }} & ={ }_{2}^{3} \mathrm{~B}_{0 \text { Predicted }} \cong 2 m_{u}+\sqrt{m_{u} m_{d}} \\
& =0.008323342076 u .
\end{aligned}
$$

The empirical energy from Figure 3, in comparison, is ${ }_{2}^{3} \mathrm{~B}_{0}=0.008285602824 u$, so that:

$$
\begin{aligned}
& { }_{2}^{3} \mathrm{~B}_{0 \text { Predicted }}-{ }_{2}^{3} \mathrm{~B}_{0} \\
& =0.008323342076 u-0.008285602824 u \\
& =0.000037739252 u .
\end{aligned}
$$

While not quite as close as (5.2) for the alpha particle, this is still a very close match to just under 4 parts in 100,000 AMU. But does this make sense in light of the outer products (4.9), (4.10)?

If we wish to write (6.1) in the manner of (5.3) and (5.4) in terms of the components of an outer tensor product $E_{A B B A}$, then referring to (4.9), we find that:

$$
\begin{aligned}
{ }_{2}^{3} \mathrm{~B}_{0 \text { Predicted }} & =(2 \pi)^{\frac{3}{2}} E_{\mathrm{P} 33 A A}=2 m_{u}+\sqrt{m_{u} m_{d}} \\
& =\sqrt{m_{u}}\left(\sqrt{m_{d}}+2 \sqrt{m_{u}}\right)
\end{aligned}
$$

So the expression $2 m_{u}+\sqrt{m_{u} m_{d}}$ in (6.1) in fact has a very natural formulation which utilizes the trace $\sqrt{m_{d}}+2 \sqrt{m_{u}}$ (AA index summation) of one of the matrices in (4.9), times a $\sqrt{m_{u}}$ taken from the 33 (or possibly 22) diagonal component of the other matrix in (4.9). The use in (6.3) of $E_{\mathrm{P}}$ from (4.9) rather than of $E_{\mathrm{N}}$ from (4.10), draws from the fact that we need the AA trace to be $\sqrt{m_{d}}+2 \sqrt{m_{u}}$, and not $\sqrt{m_{u}}+2 \sqrt{m_{d}}$ as would otherwise occur if we used (4.10). So here, the empirical data clearly causes us to use $E_{\mathrm{p}}$ from the proton matrix in (4.9) rather than $E_{\mathrm{N}}$ from the neutron matrix in (4.10). We also note that physically, ${ }^{3} \mathrm{He}$ has one more proton than neutron. This is a third data point in the Yang-Mills tensor for nuclear binding.

\section{Prediction of the Triton Nuclide Binding Energy to 3 Parts in One Million, and the Neutron minus Proton Mass Difference to 7 Parts in Ten Million}

Now we turn to the ${ }_{1}^{3} \mathrm{H}$ triton nuclide, which as shown in Figure 3, has a binding energy ${ }_{1}^{3} \mathrm{~B}_{0}=0.009105585412 u$, and as discussed following (5.4), is a "bottom to top" nuclide. As with the alpha and the helion, we use the energies from components of the outer products $E_{A B C D}$, see again (4.9) to (4.11). However, following careful trial and error consideration of all possible combinations, there is no readily-apparent combination of $m_{u}, m_{d}$, $\sqrt{m_{u} m_{d}}$ together with $m_{e}$ and factors of $(2 \pi)^{\frac{3}{2}}$ which yield a close match to well under 1 percent, to ${ }_{1}^{3} \mathrm{~B}_{0}=0.009105585412 u$, which is the observed ${ }_{1}^{3} \mathrm{H}$ binding energy.

But all is not lost, and much more is found: When studying nuclear data, there are two interrelated ways to formulate that data. First, is to look at binding energies as we have done so far. Second, is to look at mass excess. 
The latter formulation, mass excess, is very helpful when studying nuclear fusion and fission processes, and as we shall now see, it is this approach that enables us to match up the empirical binding data for the triton to the $m_{u}, m_{d}, \sqrt{m_{u} m_{d}}, m_{e}$ and factors of $(2 \pi)^{\frac{3}{2}}$ that we have already successfully employed for the deuteron, alpha, and helion. As a tremendous bonus, we will be able to derive a strictly theoretical expression for the observed, empirical difference:

$$
M(n)-M(p)={ }_{0}^{1} M-{ }_{1}^{1} M=0.001388449188 u
$$

between the free, unbound neutron mass $M(n)=$ $1.008664916000 u$ and the free, unbound proton mass $M(p)=1.007276466812 u$, see Figure 2 .

The derivation of the ${ }^{3} \mathrm{He}$ binding energy and the neutron minus proton mass difference is somewhat involved, and so is detailed in the Appendix. But the results are as follows: For the neutron minus proton mass difference, in (A15), also using (1.11), we obtain:

$$
\begin{aligned}
& {[M(n)-M(p)]_{\text {Predicted }}} \\
& =m_{u}-m_{e}-2 \sqrt{m_{\mu} m_{d}} /(2 \pi)^{\frac{3}{2}} \\
& =m_{u}-\left(3 m_{d}+2 \sqrt{m_{\mu} m_{d}}-3 m_{u}\right) /(2 \pi)^{\frac{3}{2}} \\
& =0.001389166099 u
\end{aligned}
$$

which differs from the empirical (7.1) by a mere $\mathbf{0 . 0 0 0 0 0 0 7 1 6 9 1 1 u}$, or just over seven parts per ten million! And for the ${ }^{3} \mathrm{He}$ binding energy in (A17), we use the above to help obtain:

$$
\begin{aligned}
\mathrm{B}_{0}\left({ }^{3} \mathrm{H}\right)_{\text {Predicted }} & ={ }_{1}^{3} \mathrm{~B}_{0 \text { Predicted }} \\
& =4 m_{u}-2 \sqrt{m_{\mu} m_{d}} /(2 \pi)^{\frac{3}{2}} \\
& =0.009102256308 u
\end{aligned}
$$

which differs from ${ }_{1}^{3} \mathrm{~B}_{0}=0.009105585412 u$, the empirical value in Figure 3, by merely $0.000003329104 u$, or just over 3 parts per million.

A theoretical tensor expression for (7.3) using components of an outer product $E_{A B B A}$ as in (5.3), (5.4) and (6.3), may be written as:

$$
\begin{aligned}
{ }_{1}^{3} B_{0 \text { Predicted }}= & (2 \pi)^{\frac{3}{2}}\left(E_{\mathrm{P} 2222}+E_{\mathrm{P} 2233}+E_{\mathrm{P} 3322}+E_{\mathrm{P} 3333}\right) \\
& -E_{\mathrm{P} 1122}-E_{\mathrm{P} 1133} \\
= & 4 m_{u}-2 \sqrt{m_{\mu} m_{d}} /(2 \pi)^{\frac{3}{2}}
\end{aligned}
$$

As earlier noted following (5.4), there will be some flexibility in these tensor component assignments until we develop a wider swathe of binding energies beyond the " 1 s square" and start to discern the wider patterns.

With the foregoing, we have now reached our goal of deducing precise theoretical expressions for all of the $1 \mathrm{~s}$ binding energies, solely as a function of elementary fermion masses. In the process, we have also deduced a like-expression for the neutron-proton mass difference!

From here, after consolidating our binding energy results and expressing them as mass excess in Section 8, we examine the solar fusion cycle in Section 9, including possible technological implications of these results for catalyzing nuclear fusion. In Section 10 we again focus on experimental errors as we did at the end of Section 5, and explain why (7.2) should be taken as an exact theoretical relationship with the quark masses and binding energies then slightly recalibrated.

\section{Mass Excess Predictions}

Let us now aggregate some of the results so far, as well as those in the Appendix. First of all, let us draw on (A4), and use (A14) and the neutron minus proton mass difference (7.2) to rewrite (A4) as:

$$
\begin{aligned}
{ }_{1}^{3} M_{\text {Predicted }} & =M(p)+2 M(n) \\
& -4 m_{u}+2 \sqrt{m_{\mu} m_{d}} /(2 \pi)^{\frac{3}{2}}
\end{aligned}
$$

Specifically, we have refashioned (A4) to include one proton mass and two neutron masses, because the ${ }_{1}^{3} \mathrm{H}$ triton nuclide in fact contains one proton and two neutrons. Thus, $-4 m_{u}+2 \sqrt{m_{\mu} m_{d}} /(2 \pi)^{\frac{3}{2}}$ represents a theoretical value of the mass excess of two free neutrons and one free proton with $M(p)+2 M(n)$ over the mass they possess when fused into a triton, expressed via a negative number as a fusion mass loss. This is equal in magnitude and opposite in sign to binding energy (7.3).

Similarly for helium nuclei, first we use (A5) to write:

$$
\begin{aligned}
{ }_{2}^{3} \mathrm{~B}_{0} & =2 \cdot{ }_{1}^{1} M+{ }_{0}^{1} M-{ }_{2}^{3} M \\
& =2 M(p)+M(n)-{ }_{2}^{3} M
\end{aligned}
$$

We then place ${ }_{2}^{3} M$ on the left and use (6.1) to write:

$$
{ }_{2}^{3} M=2 M(p)+M(n)-2 m_{u}-\sqrt{m_{u} m_{d}} .
$$

Here, $-2 m_{u}-\sqrt{m_{u} m_{d}}$ is the fusion mass loss for the helion, also equal and opposite to binding energy (6.1).

Next, we again use (A5) to write:

$$
\begin{aligned}
{ }_{2}^{4} \mathrm{~B}_{0} & =2 \cdot{ }_{1}^{1} M+2 \cdot{ }_{0}^{1} M-{ }_{2}^{4} M \\
& =2 \cdot M(p)+2 \cdot M(n)-{ }_{2}^{4} M
\end{aligned}
$$

Combining this with (5.1) then yields:

$$
\begin{aligned}
{ }_{2}^{4} M & =2 M(p)+2 M(n)-6 m_{u}-6 m_{d} \\
& +\frac{10 m_{d}+10 m_{u}+16 \sqrt{m_{u} m_{d}}}{(2 \pi)^{\frac{3}{2}}}+2 \sqrt{m_{u} m_{d}}
\end{aligned}
$$


The fusion mass loss for the alpha - much larger than for the other nuclides we have examined - is given by the lengthier terms after $2 M(p)+2 M(n)$. Again, this is equal and opposite to the alpha binding energy in (5.1), with terms consolidated above.

Finally, from (4.1), via (A5), it is easy to deduce for the deuteron, that:

$$
{ }_{1}^{2} M \equiv M(p)+M(n)-m_{u},
$$

with a mass loss represented simply by $-m_{u}$, again, equal and opposite the binding energy (4.1).

\section{A Theoretical Review of the Solar Fusion Cycle, and a Possible Approach to Catalyzing Fusion Energy Release}

As a practical exercise, let us now use all of the foregoing results to theoretically examine the solar fusion cycle. The first step in this cycle is (A10) for the fusion of two protons into a deuteron. It is from (A10) that we determine that an energy (A11) is released in this fusion, which energy, in light of (A13), now becomes:

$$
\operatorname{Energy}\left({ }_{1}^{1} \mathrm{H}+{ }_{1}^{1} \mathrm{H} \rightarrow{ }_{1}^{2} \mathrm{H}+e^{+}+v+\text { Energy }\right)=2 \sqrt{m_{\mu} m_{d}} /(2 \pi)^{\frac{3}{2}}=0.000451141003 u
$$

This equates to $0.420235 \mathrm{MeV}$ which is a well-known energy in solar fusion as is noted in the Appendix. The positron annihilates with an electron $e^{+}+e^{-} \rightarrow \gamma+\gamma$ to produce an additional $2 m_{e}$ worth of energy as well. The second reaction in the solar fusion cycle is:

$$
\begin{array}{r}
{ }_{1}^{2} \mathrm{H}+{ }_{1}^{1} \mathrm{H} \rightarrow{ }_{2}^{3} \mathrm{He}+\text { Energy } \\
\text { Energy }\left({ }_{1}^{2} \mathrm{H}+{ }_{1}^{1} \mathrm{H} \rightarrow{ }_{2}^{3} \mathrm{He}+\text { Energy }\right)=m_{u}+\sqrt{m_{u} m_{d}}=0.005935171976 u
\end{array}
$$

where in deuterons produced in (9.1) fuse with protons to produce helions. We write this in terms of masses as:

$$
\text { Energy }={ }_{1}^{2} M+{ }_{1}^{1} M-{ }_{2}^{3} M \text {. }
$$

The proton mass is ${ }_{1}^{1} M$, and these other two masses have already been found, respectively, in (8.6) and (8.3).

which equates to $5.528577 \mathrm{MeV}$, also a well-known energy in the study of solar fusion.

The final step in this cycle fuses two helions together to yield alpha particles plus protons, which protons then are available to repeat the cycle starting at (9.1):

$$
\begin{gathered}
\begin{aligned}
{ }_{2}^{3} \mathrm{He}+{ }_{2}^{3} \mathrm{He} \rightarrow{ }_{2}^{4} \mathrm{He}+{ }_{1}^{1} \mathrm{H}+{ }_{1}^{1} \mathrm{H}+\text { Energy } & (9.5) \\
\text { Energy }\left({ }_{2}^{3} \mathrm{He}+{ }_{2}^{3} \mathrm{He} \rightarrow{ }_{2}^{4} \mathrm{He}+{ }_{1}^{1} \mathrm{H}+{ }_{1}^{1} \mathrm{H}+\text { Energy }\right) & =2 m_{u}+6 m_{d}-4 \sqrt{m_{u} m_{d}}-\left(10 m_{d}+10 m_{u}+16 \sqrt{m_{u} m_{d}}\right) /(2 \pi)^{\frac{3}{2}} \\
& =0.013732528003 u
\end{aligned}
\end{gathered}
$$

This equates to $12.791768 \mathrm{MeV}$, which is also a well-known energy from solar fusion studies.

Now, as is well known (see, e.g. [14]), the reaction (9.4) must occur twice to produce the two ${ }_{2}^{3} \mathrm{He}$ which are input to (9.7), and the reaction (9.1) must occur twice to produce the two ${ }_{1}^{2} \mathrm{H}$ which are in turn input to (9.4). So pulling this all together from (9.1), (9.4), (9.7) and $e^{+}+e^{-} \rightarrow \gamma+\gamma$, we may express the entire solar fusion cycle in (9.8) below. In the top line below, we show in detail each energy release from largest to smallest, followed by the electron and neutrino emissions. In the second line we segregate in separate parenthesis, each contribution shown in the top line, including the neutrino mass which is virtually zero. In the third line, we consolidate terms. In the final line we use (1.11) to eliminate the electron rest mass:

$$
\begin{aligned}
& \operatorname{Energy}\left(4 \cdot{ }_{1}^{1} \mathrm{H}+2 e^{-} \rightarrow{ }_{2}^{4} \mathrm{He}+\gamma(12.79 \mathrm{MeV})+2 \gamma(5.52 \mathrm{MeV})+2 \gamma(0.42 \mathrm{MeV})+4 \gamma(e)+2 v\right) \\
& =\left(2 m_{u}+6 m_{d}-4 \sqrt{m_{u} m_{d}}-\frac{10 m_{d}+10 m_{u}+16 \sqrt{m_{u} m_{d}}}{(2 \pi)^{\frac{3}{2}}}\right)+2\left(m_{u}+\sqrt{m_{u} m_{d}}\right)+2\left(2 \frac{\sqrt{m_{\mu} m_{d}}}{(2 \pi)^{\frac{3}{2}}}\right)+4\left(m_{e}\right)+2\left(m_{v}\right) \\
& =4 m_{u}+6 m_{d}+4 m_{e}-2 \sqrt{m_{u} m_{d}}-\frac{10 m_{d}+10 m_{u}+12 \sqrt{m_{u} m_{d}}}{(2 \pi)^{\frac{3}{2}}} \\
& =4 m_{u}+6 m_{d}-2 \sqrt{m_{u} m_{d}}+\frac{2 m_{d}-22 m_{u}-12 \sqrt{m_{u} m_{d}}}{(2 \pi)^{\frac{3}{2}}}=26.733389 \mathrm{MeV}
\end{aligned}
$$


The above shows at least two things. First, the total energy of approximately $26.73 \mathrm{MeV}$ known to be released during solar fusion is expressed entirely in terms of a theoretical combination of the up and down (and optionally electron) masses, with nothing else added! This portends the ability to do the same for other types of fusion and fission, once the analysis of this paper is extended to larger nuclides $Z>2, N>2$.

Secondly, because the results throughout this paper seem to validate modeling nucleons as resonant cavities with energies released or retained based on the masses of their quark contents, this tells us how to catalyze "resonant fusion" which may make fusion technology more practical, because (9.8) tells us the precise resonances that go into releasing the total $26.73 \mathrm{MeV}$ of energy in the above. In particular, if one wanted to create an artificial "sun in a box," one would be inclined to amass a store of hydrogen, and subject that hydrogen store to gamma radiation at or near the specified discrete energies that appear in (9.8), so as to facilitate resonant cavity vibrations at or near the energies required for fusion to occur. Specifically, one would bathe the hydrogen store with gamma radiation at one or more of the following energies/frequencies in combination, some without, and some with, the Gaussian $(2 \pi)^{\frac{3}{2}}$ divisor (we convert to wavelengths via $1 \mathrm{~F}=1 /(197 \mathrm{MeV}))$ :

$$
\begin{aligned}
& 6 m_{d}=29.44 \mathrm{MeV}=6.69 \mathrm{~F} \\
& m_{u}=2.22 \mathrm{MeV}=88.56 \mathrm{~F} \\
& 2 m_{u}(\text { harmonic })=4.45 \mathrm{MeV}=44.28 \mathrm{~F} \\
& 4 m_{u}(\text { harmonic })=8.90 \mathrm{MeV}=22.14 \mathrm{~F} \\
& \sqrt{m_{u} m_{d}}=3.30 \mathrm{MeV}=59.62 \mathrm{~F} \\
& 2 \sqrt{m_{u} m_{d}}(\text { harmonic })=6.61 \mathrm{MeV}=29.81 \mathrm{~F} \\
& 4 \sqrt{m_{u} m_{d}}(\text { harmonic })=13.22 \mathrm{MeV}=14.91 \mathrm{~F} \\
& 2 m_{d} /(2 \pi)^{\frac{3}{2}}=0.62 \mathrm{MeV}=316.15 \mathrm{~F} \\
& 10 m_{d} /(2 \pi)^{\frac{3}{2}}=3.12 \mathrm{MeV}=63.23 \mathrm{~F} \\
& 10 m_{u} /(2 \pi)^{\frac{3}{2}}=1.41 \mathrm{MeV}=139.47 \mathrm{~F} \\
& 22 m_{u} /(2 \pi)^{\frac{3}{2}}=3.10 \mathrm{MeV}=63.40 \mathrm{~F} \\
& 2 \sqrt{m_{u} m_{d}} /(2 \pi)^{\frac{3}{2}}=0.42 \mathrm{MeV}=469.53 \mathrm{~F} \\
& 4 \sqrt{m_{u} m_{d}} /(2 \pi)^{\frac{3}{2}}(\text { harmonic })=0.84 \mathrm{MeV}=234.77 \mathrm{~F} \\
& 12 \sqrt{m_{u} m_{d}} /(2 \pi)^{\frac{3}{2}}(\text { harmonic })=2.52 \mathrm{MeV}=78.26 \mathrm{~F} \\
& 16 \sqrt{m_{u} m_{d}} /(2 \pi)^{\frac{3}{2}}(\text { harmonic })=3.36 \mathrm{MeV}=58.69 \mathrm{~F}
\end{aligned}
$$

In the above, we have explicitly shown each basic frequency/energy which appears in the second, third or fourth lines of (9.8) as well as harmonics that appear in (9.8). Also, one should consider frequencies based on the electron mass and its wavelength.

So, what do we learn? If the nucleons are regarded as resonant cavities and the energies at which they fuse depend on the masses of their current quarks as is made very evident by (9.8), and given the particular energies and harmonics highlighted in (9.9) and (9.10), the idea for harmonic fusion is to subject a hydrogen store to high-frequency gamma radiation proximate at least one of the frequencies (9.9), (9.10), with the view that these harmonic oscillations will catalyze fusion by perhaps reducing the amount of heat that is required. In present-day approaches, fusion reactions are triggered using heat generated from a fission reaction, and one goal would be to reduce or eliminate this need for such high heat and especially the need for any fissile trigger. That is, we at least posit the possibility-subject of course to laboratory testing to confirm feasibility - that applying the harmonics (9.9), (9.10) to a hydrogen store can catalyze fusion better than known methods, with less heat and ideally little or no fission trigger required.

Of course, these energies in (9.9), (9.10) are very high, and aside from the need to produce this radiation via known methods such as, but not limited to, Compton backscattering and any other methods which are known at present or may become known in the future for producing gamma radiation, it would also be necessary to provide substantial shielding against the health effects of such radiation. The highest energy/smallest wavelength component, $\quad 6 m_{d}=29.44 \mathrm{MeV}=6.69 \mathrm{~F}$, is extremely energetic and would be very difficult to shield (and to produce), but this resonance arises from (9.8) which is for the final ${ }_{2}^{3} \mathrm{He}+{ }_{2}^{3} \mathrm{He} \rightarrow{ }_{2}^{4} \mathrm{He}+{ }_{1}^{1} \mathrm{H}+{ }_{1}^{1} \mathrm{H}+$ Energy portion of the solar fusion cycle. If one were to forego this portion of the fusion cycle and focus only on catalyzing ${ }_{1}^{1} \mathrm{H}+{ }_{1}^{1} \mathrm{H} \rightarrow{ }_{1}^{2} \mathrm{H}+e^{+}+v+$ Energy to fuse protons into deuterons, then the only needed resonance is

$$
2 \sqrt{m_{u} m_{d}} /(2 \pi)^{\frac{3}{2}}=0.42 \mathrm{MeV}=469.53 \mathrm{~F} .
$$

Not only is this easiest to produce because its energy is the lowest of all the harmonics in (9.9) and (9.10), but it is the easiest to shield and the least harmful to humans.

Certainly, a safe, reliable and effective method and associated hardware for producing energy via fusing protons into deuterons via reaction (9.1), and perhaps further fusing protons and deuterons into helions as in (9.4), by introducing at least one of the harmonics (9.9), (9.10) into a hydrogen store perhaps in combination with other known fusion methods, while insufficient to create the "artificial sun" modeled above if one foregoes the 
final alpha production in (9.7), would nonetheless represent a welcome, practical addition to sources of energy available for all forms of peaceful human endeavor.

\section{Recalibration of Masses and Binding Energies via an Exact Relationship for the Neutron minus Proton Mass Difference}

At the end of Section 5, we briefly commented on experimental errors. As between the alpha particle and the deuteron, we determined it was more sensible to associate the binding energy of the deuteron precisely with the mass of the up quark, thus making the theoretically-predicted alpha binding energy a close but not exact match to its empirically observed value, rather than vice versa. But the prediction in (7.2) for the neutron minus proton mass difference to just over 7 parts in ten million is a very different matter. This is even more precise by half an order of magnitude than the alpha mass prediction, and given the fundamental nature of the relationship for $M(n)-M(p)$ which is central to beta-decay, we now argue why (7.2) should be taken as an exact relationship with all other relationships recalibrated accordingly, so that now the up quark mass will still be very close to the deuteron binding energy, but will no longer be exactly equal to this energy.

First of all, as just noted, the $M(n)-M(p)$ mass difference is the most precisely predicted relationship of all the relationships developed above, to under one part per million $A M U$. Second, we have seen that all the other nuclear binding energies we have predicted are close approximations, but not exact, and would expect that this inexactitude will grow larger as we consider even heavier nuclides, see, for example, ${ }^{8} \mathrm{Be}$ as discussed in Figures 7 and 8. So, rhetorically speaking, why should the deuteron be so "special," as opposed to any other nuclide, such that it gets to have an "exact" relation to some combination of elementary fermion masses while all the other nuclides do not? Yes, the deuteron should come closest to the theoretical prediction (namely the up mass) of all nuclides, because it is the smallest composite nuclide. Closer than all other nuclides, but still not exact. After all, even the $A=2$ deuteron should suffer from "large $A=Z$ $+N$ " effects even if only to the very slightest degree of parts per ten million. Surely it should suffer these effects more than the $A=1$ proton or neutron.

Third, if this is so, then we gain a new footing to be able to consider how the larger nuclides differ from the theoretical ideal, because even for this simplest $A=2$ deuteron nuclide, we will already have a preciselyknown deviation of the empirical data from the theoreticcal prediction, which we may perhaps be able to extrapolate to larger nuclides for which this deviation cer- tainly becomes enhanced. That is, the deviations between predicted and empirical binding data for all nuclides becomes itself a new data set to be studied and hopefully explained, thus perhaps providing a foundation to theoreti- cally eliminate even this remaining deviation.

Fourth, in a basic sense, the deuteron, which is one proton fused to one neutron, has a mass which is a measure of "neutron plus proton," while $M(n)-M(p)$ is a measure of "neutron minus proton." So we are really faced with a question of what gets to be exact and what must be only approximate: $n+p$, or $n-p$ ? Seen in this light, $M(n)-M(p)$ measures an energy feature of neutrons and protons in their native, unbound states, as separate and distinct entities, and thus characterizes these elemental nucleons in their purest form. In the deuteron, by contrast, we have a two-body system which is lesspure. So if we must choose between one or the other, we should choose $M(n)-M(p)$ to be exact relationship, with the chips falling where they may for all other relationships, including the deuteron binding energy. Now, the deuteron binding energy is relegated to the same "approximate" status as that of all other compound polynuclides, and only the proton and neutron as distinct mono-nuclides get to enjoy "exact" status.

Let us therefore do exactly that. Specifically, for the reasons given above, we now abandon our original postulate that the up quark mass is exactly equal to the deuteron binding energy, and in its place we substitute the postulate that (7.2) is an exact relationship, period. That is, we now define, by substitute postulate, that the exact relationship which drives all others, is:

$$
\begin{aligned}
& {[M(n)-M(p)]_{\text {Observed }}} \\
& =0.001388449188 u \\
& \equiv m_{u}-\left(3 m_{d}+2 \sqrt{m_{\mu} m_{d}}-3 m_{u}\right) /(2 \pi)^{\frac{3}{2}} \\
& =[M(n)-M(p)]_{\text {Predicted }}
\end{aligned}
$$

Then, we modify all the other relationships accord- ingly.

The simplest way make this adjustment is to modify the original postulate (4.1) to read:

$$
m_{u} \equiv{ }_{1}^{2} \mathrm{~B}_{0}+\varepsilon=0.002388170100 u+\varepsilon,
$$

and to then substitute this into (10.1) with $\varepsilon$ taken as very small but unknown. This is most easily solvable numerically, and it turns out that $\varepsilon=-0.000000830773 u$, which is just over 8 parts in ten million $u$. That is, substituting $\varepsilon=-0.000000830773 u$ into (10.2), then using (1.11) to derive the down quark mass, then substituting all of that into (10.1), will make (10.1) exact through all twelve decimal places (noting that experimental errors are in the last two places).

As a consequence, the following critical mass/energies 
developed earlier become nominally adjusted starting at the sixth decimal place in AMU, and now become (contrast (4.1), (4.3), (4.4), (4.5) and (4.6) respectively):

$$
\begin{aligned}
& m_{u}=0.002387339327 u, \\
& m_{d}=0.005267312526 u, \\
& \sqrt{m_{u} m_{d}}=0.003546105236 u, \\
& B_{\mathrm{P}}=2 m_{u}+m_{d}-\left(m_{d}+4 \sqrt{m_{u} m_{d}}+4 m_{u}\right) /(2 \pi)^{\frac{3}{2}} \\
& =0.008200606481 u \\
& B_{\mathrm{N}}=2 m_{d}+m_{u}-\left(m_{u}+4 \sqrt{m_{u} m_{d}}+4 m_{d}\right) /(2 \pi)^{\frac{3}{2}} \\
& =0.010531999771 u
\end{aligned}
$$

Additionally, this will slightly alter the binding energies that were predicted earlier. The new results are as follows (contrast (5.1), (6.1) and (7.3) respectively):

$$
\begin{aligned}
& { }_{2}^{4} \mathrm{~B}_{0 \text { Predicted }}=0.030373002032 u, \\
& { }_{2}^{3} \mathrm{~B}_{0 \text { Predicted }}=0.008320783890 u . \\
& { }_{1}^{3} \mathrm{~B}_{0 \text { Predicted }}=0.009099047078 u .
\end{aligned}
$$

and, via (10.3) and this adjustment of masses,

$$
{ }_{1}^{2} \mathrm{~B}_{0 \text { Predicted }}=m_{u}=0.002387339327 u .
$$

In (10.11), we continue to regard the predicted deuteron binding energy ${ }_{1}^{2} \mathrm{~B}_{0 \text { Predicted }}$ to be equal to the mass of the up quark, but because the mass of the up quark has now been slightly changed because of our substitute postulate, the observed energy, which is ${ }_{1}^{2} \mathrm{~B}_{0}=$ $0.002388170100 u$, will no longer be exactly equal to the predicted energy (10.11). Rather, we will now have ${ }_{1}^{2} \mathrm{~B}_{0} \neq{ }_{1}^{2} \mathrm{~B}_{0 \text { Predicted }}$, with a difference of less than one part per million AMU. The precise, theoretical exactitude now belongs to the $M(n)-M(p)$ difference in (10.1). As a bonus, the up and down quark masses now become known to ten-digit precision in $A M U$, with experimental errors in the 11th and 12th digits, which is inherited from the precision with which the electron, proton and neutron masses are known.

One other point is very much worth noting. With an entirely theoretical, exact expression now developed for the neutron minus proton mass difference via (10.1), we start to target the full, dressed proton and neutron masses themselves. Specifically, it would be extremely desirable to be able to specify the proton and neutron masses as a function of the elementary up, down, and electron fermion masses, as we have here with binding energies. Fundamentally, by elementary algebraic principles, taking each of the proton and neutron masses as an unknown, we can deduce these masses if we have can find two independent equations, one of which contains an exact expression related to the sum of these masses, and the other which contains an exact expression related to the difference of these masses. Equation (10.1) achieves the first half of this objective: for the first time, we now have an exact theoretical expression for the difference between these masses. But we still lack an independent expression related to their sum.

Every effort should now be undertaken to find another relationship related to the sum of these masses. In all likelihood, that relationship, which must inherently explain the natural ratio just shy of 1840 between the masses of the nucleons and the electron, and/or similar ratios of about 420 and 190 involving the up and down masses, will need to emerge from an examination of the amended t'Hooft Lagrangian terms in (3.10) which we have not yet explored, particularly those terms which involve the vacuum $\Phi$. While analyzing binding energies and mass excess and nuclear reactions as we have done here is a very valuable exercise, the inherent limitation is that all of these analyses involve differences. What is needed to obtain the "second" of the desired two independent equations, are sums, not differences (Note: the author lays the GUT foundation for, and then tackles this very problem, in two separate papers published in this same special issue of JMP).

\section{Summary and Conclusion}

Summarizing our results here, we now have the following theoretical predictions for the binding energies in Figure 3, with isobar lines shown, and with equation numbers for result referenced for convenience: see Figure 9.

The mass loss (negative mass excess) discussed in Section 8 which was very helpful to the exercise of examining the solar fusion cycle in Section 9, is simply the negative (positive) of what is shown in Figure 9. Having just considered the $M(n)-M(p)$ mass difference, it is useful to also look at the difference between the ${ }^{3} \mathrm{H}$ and ${ }^{3} \mathrm{He}$ isobars, $A=3$ in the above. Given that ${ }^{3} \mathrm{He}$ is the stable nuclide and that ${ }^{3} \mathrm{H}$ undergoes $\beta^{-}$decay into ${ }^{3} \mathrm{He}$, we may calculate the predicted difference in binding energies to be:

$$
\begin{aligned}
{\left[{ }_{2}^{3} \mathrm{~B}_{0}-{ }_{1}^{3} \mathrm{~B}_{0}\right] \text { Predicted } } & =-2 m_{u}+\left(1+\frac{2}{(2 \pi)^{\frac{3}{2}}}\right) \sqrt{m_{u} m_{d}} \\
& =-0.000778263189 u
\end{aligned}
$$

The empirical difference $-0.000819982588 u$ differs from the predicted difference by $0.000041719399 u$. It is helpful to contrast the above to (the negative of) (10.1) which represents the most elementary $\beta^{-}$decay of a neutron into a proton. Similar calculations may be carried out as between the isotopes and isotones in Figure 9.

The numerical values of these theoretical binding en- 
ergies in Figure 9, in AMU, using the recalibrated (10.8) through (10.11), are now predicted to be: see Figure 10.

These theoretical predictions should be carefully compared to the empirical values in Figure 3. Indeed, subtracting each entry in Figure 3 from each entry in Figure 10, we summarize our results for all of the $1 \mathrm{~s}$ nuclides in Figure 11.

Figure 11 shows how much each predicted binding energy differs from observed empirical binding energies. As has been reviewed, every one of these predictions is accurate to under four parts in 100,000 AMU $\left({ }^{3} \mathrm{He}\right.$ has the largest difference). Specifically: we have now used the thesis that baryons are resonant cavity Yang-Mills magnetic monopoles with binding energies reflective of their current quark masses to predict the binding energies of the ${ }^{4} \mathrm{He}$ alpha to under four parts in one million, of the ${ }^{3} \mathrm{He}$ helion to under four parts in 100,000 and of the ${ }^{3} \mathrm{H}$ triton to under seven parts in one million. Of special import, we have exactly related the neutron minus proton mass difference - which is central to beta decay - to the up and down quark masses. This in turn enables us via the substitute postulate of Section 10 to predict the bind- ing energy for the ${ }^{2} \mathrm{H}$ deuteron most precisely of all, to just over 8 parts in ten million.

These energies as well as the neutron minus proton mass difference do not appear to have ever before been theoretically explained with such accuracy, and each of the foregoing energy predictions is mutually-independent from all the others. So even if any one prediction is thought to be nothing more than coincidence, the odds against five independent predictions on the order of 1 part in $10^{5}$ or better being mere coincidence exceed $10^{25}$ to 1 . This is not mere coincidence!

This leads to the conclusion that the underlying thesis that baryons generally, and neutrons and protons especially, are resonant cavity Yang-Mills magnetic monopoles with binding energies determined by their current quark masses, provides the strongest theoretical explanation to date of what baryons are, and of how protons and neutrons confine their quarks and bind together into composite nuclides. The theory of nuclear binding first developed in [1] and further amplified here, establishes a basis for finally "decoding" the abundance of known data regarding nuclear masses and binding energies, and by

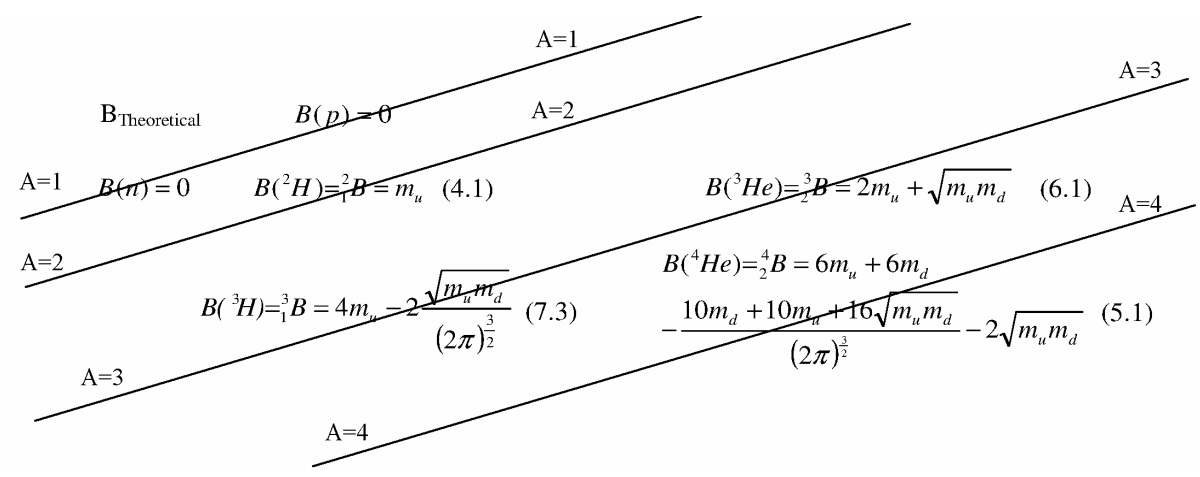

Figure 9. Binding energies $\left({ }_{z}^{A} B_{0}\right)$ of 1s nuclides (Theoretical, AMU).

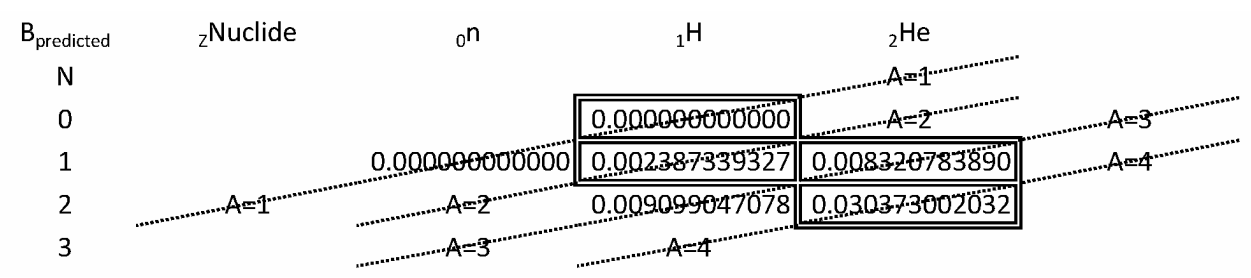

Figure 10. Binding energies $\left({ }_{z}^{A} B_{0}\right)$ of 1s nuclides (Predicted, AMU).

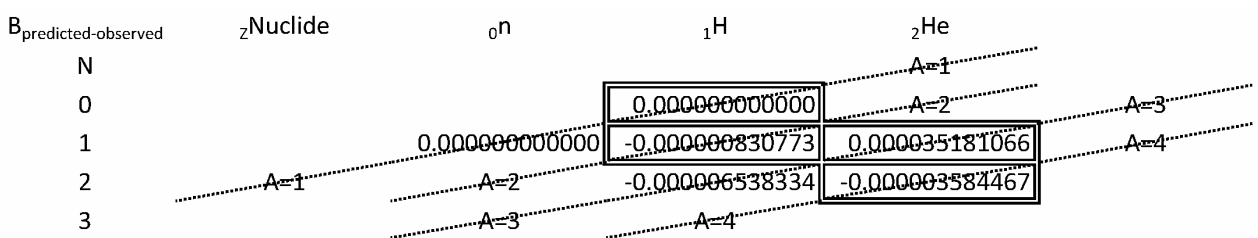

Figure 11. Predicted minus observed binding energies $\left({ }_{z}^{A} B_{0}\right)$ of 1s nuclides (AMU). 
viewing the proton and neutron as resonant cavities, may lay the foundation for technologically realizing the theoretical promise of nuclear fusion.

Finally, because nucleons are now understood to be non-Abelian magnetic monopoles, this also means that atoms themselves comprise core magnetic charges (nucleons) paired with orbital electric charges (electrons), with the periodic table itself thereby revealing an electric/magnetic symmetry of Maxwells' equations which has heretofore gone unrecognized in the 140 years since Maxwell first published his Treatise on Electricity and Magnetism.

\section{REFERENCES}

[1] J. R. Yablon, "Why Baryons Are Yang-Mills Magnetic Monopoles," Hadronic Journal, Vol. 35, No. 4, 2012, pp. 401-468.

http://www.hadronicpress.com/issues/HJ/VOL35/HJ-35-4 .pdf

[2] G. t'Hooft, "Magnetic Monopoles in Unified Gauge Theories," Nuclear Physics B, Vol. 79, 1974, pp. 276284. doi:10.1016/0550-3213(74)90486-6

[3] A. M. Polyakov, "Particle Spectrum in the Quantum Field Theory," JETP Letters, Vol. 20, 1974, pp. 194-195.
[4] H. C. Ohanian, "What Is Spin?" American Journal of Physics, Vol. 54, No. 6, 1986, pp. 500-505. doi:10.1119/1.14580

[5] F. Halzen and A. D. Martin, "Quarks and Leptons: An Introductory Course in Modern Particle Physics," John Wiley \& Sons, Hoboken, 1984.

[6] T.-P. Cheng and L.-F. Li, "Gauge Theory of Elementary Particle Physics," Oxford, 1984.

[7] S. Weinberg, "The Quantum Theory of Fields, Volume II, Modern Applications," Cambridge, 1996.

[8] G. E. Volovok, "The Universe in a Helium Droplet," Clarendon Press, Oxford, 2003.

[9] H. Georgi and S. Glashow, "Unity of All ElementaryParticle Forces," Physical Review Letters, Vol. 32, 1974, p. 438. doi:10.1103/PhysRevLett.32.438

[10] http://www.tau.ac.il/ elicomay/emc.html

[11] J. Beringer, et al., (Particle Data Group), "PR D86, 010001," 2012. http://pdg.lbl.gov

[12] http://physics.nist.gov/cuu/Constants/index.html

[13] http://en.wikipedia.org/wiki/Table_of_nuclides_(complet e)

[14] http://library.thinkquest.org/3471/fusion.html 


\section{Appendix-Detailed Derivation of the Triton Nuclide Binding Energy and the Neutron minus Proton Mass Difference}

To derive the triton binding energy, we start by considering a hypothetical process to fuse a ${ }_{1}^{1} \mathrm{H}$ nucleus (proton) with a ${ }_{1}^{2} \mathrm{H}$ nucleus (deuteron) to produce a ${ }_{1}^{3} \mathrm{H}$ nucleus (triton), plus whatever by-products emerge from the fusion. Because the inputs ${ }_{1}^{1} \mathrm{H}$ and ${ }_{1}^{2} \mathrm{H}$ each have a charge of +1 , and the output ${ }_{1}^{3} \mathrm{H}$ also has a charge of +1 , a positron will be needed to carry off the additional electric charge, and this will need to be balanced with a neutrino. Of course, there will be some fusion energy released. So in short, the fusion reaction we now wish to study is:

$$
{ }_{1}^{1} \mathrm{H}+{ }_{1}^{2} \mathrm{H} \rightarrow{ }_{1}^{3} \mathrm{H}+e^{+}+v+\text { Energy }
$$

The question: how much energy is released?

As we can see, this process includes a $\beta^{+}$decay. If we neglect the neutrino mass $m_{v} \cong 0$, and since $m_{e^{+}}=m_{e}$, we can reformulate (A1) using the nuclide masses in Figure 2, as the empirical relationship:

$$
\text { Energy }={ }_{1}^{1} M+{ }_{1}^{2} M-{ }_{1}^{3} M-m_{e}=0.004780386215 u \text { (A2) }
$$

If we then return to our "toolkit" (4.11), we see that $2 m_{u}=0.004776340200 u$. The difference:

$$
\begin{aligned}
\text { Energy }-2 m_{u} & =0.004780386215 u \\
& -0.004776340200 u \\
& =-0.000004046015 u
\end{aligned}
$$

is four parts per million! So, we now regard Energy $\cong 2 m_{u}$ to be very close relationship to the empirical data for the reaction (A1) with energy release (A2). For the deuteron, alpha and helion, our toolkit matched up to a binding energy. But for the triton, in contrast, our toolkit instead matched up to a fusion-release energy. A new player in this mix, which has not heretofore become directly involved in predicting binding energies, is the electron rest mass in (A2). So, based on (A3), we set Energy $=2 m_{u}$, and then rewrite (A2), using ${ }_{1}^{1} M=M(p)$, as:

$$
{ }_{1}^{3} M_{\text {Predicted }}=M(p)+{ }_{1}^{2} M-2 m_{u}-m_{e} .
$$

Now let's reduce. To translate between Figures 2 and 3, we of course used:

$$
{ }_{Z}^{A} \mathrm{~B}_{0}=Z \cdot{ }_{1}^{1} M+N \cdot{ }_{0}^{1} M-{ }_{Z}^{A} M
$$

which relates observed binding energy $\mathrm{B}_{0}$ in general, to nuclear mass/weight $M$ in general. So we now use (A5) specifically for ${ }_{1}^{3} \mathrm{~B}_{0}$ and combine this with (A4) using ${ }_{0}^{1} M=M(n)$, to write:

$$
\begin{aligned}
{ }_{1}^{3} \mathrm{~B}_{\text {0Predicted }} & =1 \cdot{ }_{1}^{1} M+2 \cdot{ }_{0}^{1} M-{ }_{1}^{3} M \\
& =2 M(n)-{ }_{1}^{2} M+2 m_{u}+m_{e}
\end{aligned}
$$

Then, to take care of the remaining deuteron mass ${ }_{1}^{2} M$ in the above, we use (A5) a second time, now for ${ }_{1}^{1} \mathrm{~B}_{0}$ :

$$
\begin{aligned}
{ }_{1}^{2} \mathrm{~B}_{0 \text { Predicted }} & ={ }_{1}^{1} M+{ }_{0}^{1} M-{ }_{1}^{2} M \\
& =M(p)+M(n)-{ }_{1}^{2} M
\end{aligned}
$$

We then combine (A7) rewritten in terms of ${ }_{1}^{2} M$, with (A6) to obtain:

$$
\begin{aligned}
{ }_{1}^{3} \mathrm{~B}_{0 \text { Predicted }} & =M(n)-M(p) \\
& +{ }_{1}^{2} \mathrm{~B}_{0 \text { Predicted }}+2 m_{u}+m_{e}
\end{aligned}
$$

Now all we need is ${ }_{1}^{2} \mathrm{~B}_{0 \text { Predicted }}$. But this is just the deuteron binding energy in (5.4). So a final substitution of ${ }_{1}^{2} \mathrm{~B}_{0 \text { Predicted }}=m_{u}$ into (A8) yields:

$$
{ }_{1}^{3} \mathrm{~B}_{0 \text { Predicted }}=M(n)-M(p)+3 m_{u}+m_{e} .
$$

So now, we do have a prediction for the triton binding energy, and it does include the electron rest mass, but it also includes the difference (7.1) between the free neutron and proton masses. It would be highly desirable for many reasons beyond the present exercise to also express this on a completely theoretical basis.

To do this, we repeat the analysis just conducted, but now, we fuse two ${ }_{1}^{1} \mathrm{H}$ nuclei (protons) into a single ${ }_{1}^{2} \mathrm{H}$ nucleus (deuteron). Analogously to (A1), we write:

$$
{ }_{1}^{1} \mathrm{H}+{ }_{1}^{1} \mathrm{H} \rightarrow{ }_{1}^{2} \mathrm{H}+e^{+}+v+\text { Energy, }
$$

and again ask, how much energy? This fusion, it is noted, is the first step of the process by which the sun and stars produce energy, and is the simplest of all fusions, so is interesting from a variety of viewpoints.

As in (A2), we first reformulate (A10) using the nuclide masses in Figure 2, as the empirical:

$$
\begin{aligned}
\text { Energy } & ={ }_{1}^{1} M+{ }_{1}^{1} M-{ }_{1}^{2} M-m_{e} \\
& =2 M(p)-{ }_{1}^{2} M-m_{e} \\
& =0.000451141003 u
\end{aligned}
$$

As a point of reference, this is equivalent to 0.420235 $\mathrm{MeV}$, which will be familiar to anybody to who has studied hydrogen fusion. As before, we pore over the "toolbox" in (4.11), including $(2 \pi)^{\frac{3}{2}}$ divisors, to discover that $2 \sqrt{m_{\mu} m_{d}} /(2 \pi)^{\frac{3}{2}}=0.000450424092 u$. Once again, we see a very close match, specifically:

$$
\begin{aligned}
& \text { Energy }-2 \sqrt{m_{\mu} m_{d}} /(2 \pi)^{\frac{3}{2}} \\
& =0.000451141003 u-0.000450424092 u \\
& =0.000000716911 u
\end{aligned}
$$

Here, the match is to just over 7 parts in ten million, and it is the closest match yet! So we take this too to be a 
meaningful relationship, and use this to rewrite (A11) as:

$$
2 \sqrt{m_{\mu} m_{d}} /(2 \pi)^{\frac{3}{2}}=2 M(p)-{ }_{1}^{2} M-m_{e} .
$$

Now we need to reduce this expression. First, using (4.1), namely ${ }_{1}^{2} \mathrm{~B}_{0}=m_{u}$, we write (A7) as:

$$
{ }_{1}^{2} M=M(p)+M(n)-m_{u} .
$$

Then we combine (A14) with (A13) and rearrange, and also use (1.11), to obtain the prediction:

$$
\begin{aligned}
& {[M(n)-M(p)]_{\text {Predicted }}} \\
& =m_{u}-m_{e}-2 \sqrt{m_{\mu} m_{d}} /(2 \pi)^{\frac{3}{2}} \\
& =m_{u}-\left(3 m_{d}+2 \sqrt{m_{\mu} m_{d}}-3 m_{u}\right) /(2 \pi)^{\frac{3}{2}} \\
& =0.001389166099 u
\end{aligned}
$$

This is an extremely important relationship relating the observed difference (7.1) between the neutron and proton mass $M(n)-M(p)=0.001388449188 u$ solely to the up and down (and optionally electron) rest masses. This is useful in a wide array of circumstances, especially between nuclear isobars (along the diagonal lines of like- $A$ which are shown in the figures here) which by definition convert into one another via beta decay. Comparing (A15) with (7.1), we see that:

$$
\begin{aligned}
& {[M(n)-M(p)]_{\text {Predicted }}-[M(n)-M(p)]_{\text {Observed }}} \\
& =0.001389166099 u-0.001388449188 u \\
& =0.000000716911 u
\end{aligned}
$$

This is the exact same degree of accuracy, to just over 7 parts in ten million AMU, which we saw in (A12). So this is yet another relationship matched very closely by empirical data.

Because of this, we now take (A15) to be a meaningful relationship, and use this in (A9) to write:

$$
\begin{aligned}
\mathrm{B}_{0}\left({ }^{3} \mathrm{H}\right)_{\text {Predicted }} & ={ }_{1}^{3} \mathrm{~B}_{0 \text { Predicted }} \\
& =4 m_{u}-2 \sqrt{m_{\mu} m_{d}} /(2 \pi)^{\frac{3}{2}} \\
& =0.009102256308 u
\end{aligned}
$$

As a result, we finally have a theoretical expression for the binding energy of the triton, totally in terms of the up and down quark masses. The empirical value ${ }_{1}^{3} \mathrm{~B}_{0}=$ $0.009105585412 u$ is shown in Figure 3, and doing the comparison, we have:

$$
\begin{aligned}
{ }_{1}^{3} \mathrm{~B}_{0 \text { Predicted }}-{ }_{1}^{3} \mathrm{~B}_{0} & =0.009102256308 u \\
& -0.009105585412 u \\
& =-0.000003329104 u
\end{aligned}
$$

We see that this result is accurate to just over three parts in one million AMU! 